\title{
Nueva serie de dataciones radiocarbónicas sobre hueso humano para el dolmen de Los Zumacales (Simancas, Valladolid). Reflexiones sobre la temporalidad del fenómeno megalítico en la Meseta Norte*
}

\author{
New series of radiocarbon data on human bone for Zumacales dolmen (Simancas, \\ Valladolid). Thinking about the temporality of the megalithic phenomenon in the Spanish \\ Northern Meseta
}

\section{Angélica Santa Cruz del Barrio ${ }^{a}$, Rodrigo Villalobos García ${ }^{\mathrm{b}}$ y Germán Delibes de Castro ${ }^{\mathrm{a}}$}

\begin{abstract}
RESUMEN
Se analiza la secuencia temporal funeraria de un monumento megalítico de la Meseta Norte, el dolmen de Los Zumacales (Simancas, Valladolid), fechado anteriormente en torno a la primera mitad del IV milenio cal AC. La secuencia se basa en 12 nuevas dataciones radiocarbónicas por AMS obtenidas mediante huesos humanos, seleccionadas a partir del número mínimo de individuos representados en el osario. El modelado bayesiano de las fechas define un marco temporal de uso entre $3846-3756$ y $3627-3550$ cal AC $(1 \sigma)$, coincidente con las primeras series funerarias megalíticas regionales. La concentración de la mayoría de las dataciones del IV milenio cal AC revela que la utilización del dolmen fue probablemente más intensa en su primera etapa. Además, el análisis ha identificado un individuo depositado al inicio del Calcolítico, algo que, sin dejar de considerar que la fecha sea errónea, supondría el reconocimiento de una reutilización prehistórica de la que nada se conocía hasta ahora.
\end{abstract}

\begin{abstract}
This article analyses the funerary sequence of a megalithic monument in the Northern Meseta, "Los Zumacales" dolmen (Simancas, Valladolid). This site was previously dated to around the first half of the $4^{\text {th }}$ millennium cal BC. Now, twelve radiocarbon dates by AMS, obtained from human bones, selected among the minimum number of individuals
\end{abstract}

represented in the ossuary, are presented. The Bayesian modeling of these new dates offers a chronological framework

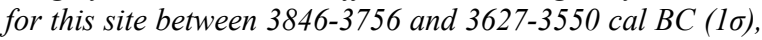
which overlaps with the first regional megalithic funerary series. It has also been possible to detect that most of the dates are concentrated in the first third of the $4^{\text {th }}$ millennium cal $B C$, revealing a probably more intense use in its early stage. The analysis has also made it possible to identify an individual deposited at the beginning of the Chalcolithic period, something which, while considering the possibility of an incorrect date, would mean the recognition of a later reuse not previously documented.

Palabras clave: Megalitismo; Prácticas funerarias; Meseta norte española; Neolítico Final; Calcolítico Inicial; Datación radiocarbónica; Modelado bayesiano; Antropología física.

Key words: Megalithic phenomenon; Mortuary practices; Spanish Northern Meseta; Late Neolithic; Early Copper Age; Radiocarbon dating; Bayesian modeling; Physical anthropology.

\section{INTRODUCCIÓN}

El fenómeno megalítico de la Meseta Norte espanola es una parcela de estudio con una tradición historiográfica de casi un siglo (p. ej. Morán 1931). Sin embargo, la época dorada de los estudios sobre el tema

* Este trabajo se enmarca en el proyecto "La neolitización del Valle Amblés (Ávila): las vías pecuarias y los marcadores territoriales como elementos articuladores del proceso" (PGC2018-099689-B-I00, Proyectos de I+D de "Generación de Conocimiento", Programa Estatal de Generación de Conocimiento y Fortalecimiento Científico y Tecnológico del Sistema de I+D+I Ministerio de Ciencia, Innovación y Universidades.

a Dpto. Prehistoria, Arqueología, Antropología Social y Ciencias y Técnicas Historiográficas. Universidad de Valladolid. Plaza del Campus Universitario s/n. 47011 Valladolid. Correos e.: angelica.santa-cruz@uva.es https://orcid.org/0000-0001-6186-0328; delibes@fyl.uva.es https://orcid.org/0000-0002-5553-6414

b Cuerpo de Profesores de Enseñanza Secundaria. Gobierno de Cantabria. Correo e.: rodrigovillalobosgarcia@gmail.com https://orcid.org/0000-0002-9064-1295

Recibido 27-IX-2019; aceptado 10-XII-2019.

Copyright: (C) 2020 CSIC. Este es un artículo de acceso abierto distribuido bajo los términos de la licencia de uso y distribución "Creative Commons Reconocimiento 4.0 Internacional" (CC BY 4.0) 
fueron los años 1980 y 1990. Se desarrollaron modernas intervenciones en cerca de medio centenar de dólmenes como los monumentos neolíticos de las penillanuras salmantinas (Delibes y Santonja 1986), de la Lora burgalesa (Delibes et al. 1993), de los altiplanos de Medinaceli (Rojo et al. 2015) o de las tierras sedimentarias del Valle Medio del Duero en las provincias de Palencia (Zapatero 2012), Zamora (Palomino 1990) y Valladolid (Delibes y Etxeberría 2002; Alonso Díez et al. 2015). La mayor parte de las investigaciones de entonces se limitaron al estudio tipológico de arquitectura y ajuares, pero con el tiempo se han ido ampliando provechosamente a la antropología física (Delibes 1995), el ADN antiguo (Szécsényi-Nagy et al. 2017), la geoestadística (Moreno 2004) o la arqueometría (Martín Gil et al. 1995; Villalobos et al. 2018). Un resultado de este proceso es la evolución de las interpretaciones sobre la sociedad megalítica que ha pasado de ser vista inicialmente como igualitaria (Delibes y Santonja 1986: 185) a percibirse, conforme se iban implementando nuevos estudios, como incipientemente desigual (Delibes 1995; Rojo et al. 2005; Guerra et al. 2009).

Ya en aquella época dorada, las contadas dataciones radiocarbónicas realizadas (Delibes 1984) permitieron no solo bosquejar a grandes rasgos la cronología del horizonte megalítico normeseteño sino también reconocer con cierto fundamento fases de construcción, uso, abandono, reutilización, etc. de los monumentos (p. ej. Delibes y Rojo 1997; Rojo et al. 2005). La naturaleza de los enterramientos colectivos constituye un indudable acicate para estos estudios relativos a las dinámicas de uso de la tumba, ya que los sepulcros pudieron amortizarse durante un breve uso o utilizarse durante periodos más prolongados, de muchas generaciones, etc. Un gran escollo para dilucidar tales incógnitas es el hecho de que los osarios suelen presentarse revueltos, salvo en contadas excepciones. Sólo recientemente el abaratamiento del precio de los análisis y el aumento de la precisión de las mediciones radiocarbónicas han inducido a plantearse la datación de todos los individuos recuperados en monumentos megalíticos para así conocer con detalle su trayectoria. En esa línea, el presente artículo se centra en el "redondil" de Los Zumacales (Simancas, Valladolid) (Fig. 1), un singular megalito de la Meseta Norte española, con el objetivo de mostrar los resultados y realizar una lectura crítica de las fechas obtenidas para la totalidad del número mínimo de individuos (NMI), procedentes de la excavación allí realizada hace más de tres décadas.

\section{EL SEPULCRO MEGALÍTICO DE LOS ZUMACALES, EN SIMANCAS}

El dolmen de Simancas, como se le conoce comúnmente, fue descubierto a mediados del siglo pasado (Romón 1960). En realidad es un monumento megalítico poco convencional cuyas losas camerales, en vez de disponerse hincadas como los ortostatos de los dólmenes canónicos, se presentan acostadas, a modo de basamento o solera de unas paredes hoy desaparecidas (Fig. 2). La estructura es muy similar a la documen-

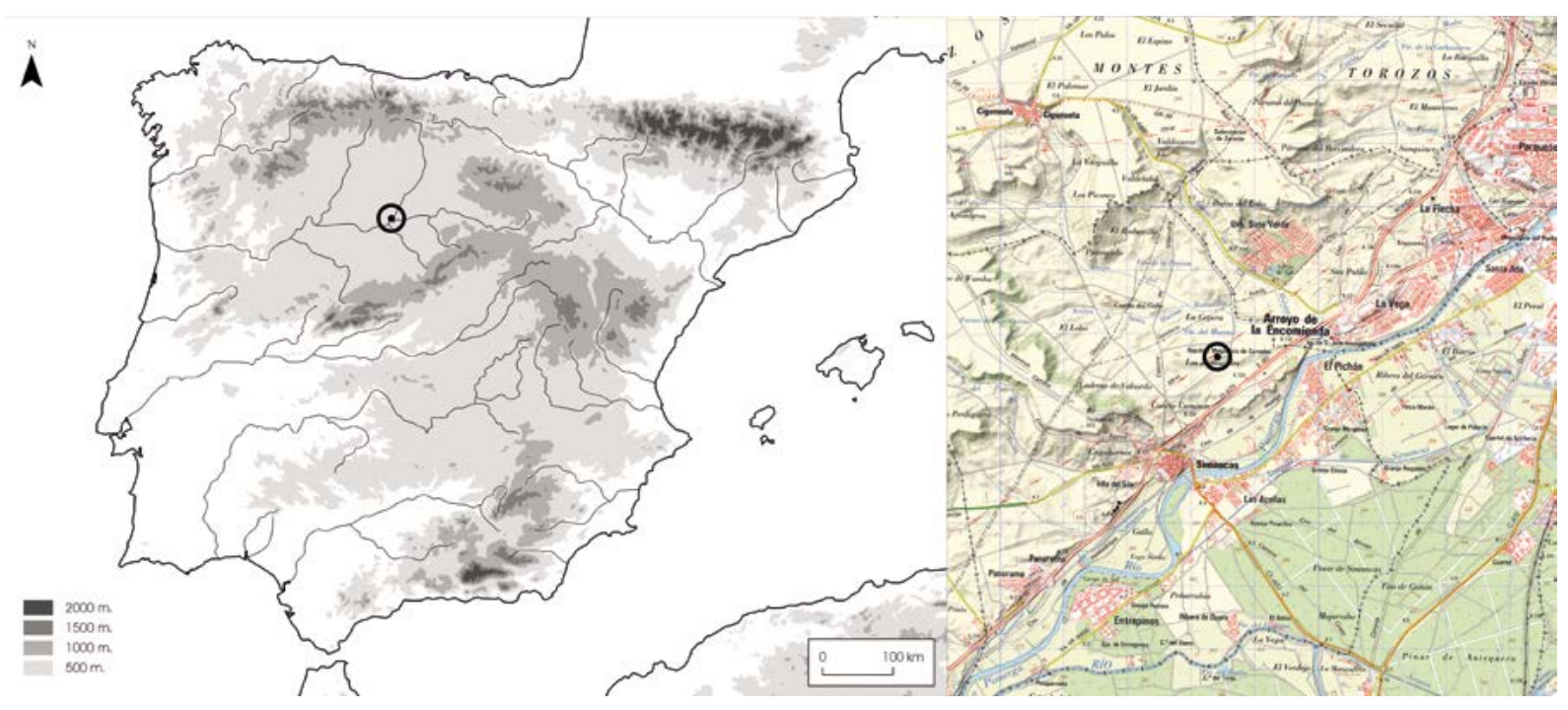

Fig. 1. Localización del dolmen de Los Zumacales (Simancas, Valladolid) en la península ibérica y su entorno inmediato (Hoja 374-1 Mapa topográfico Nacional 1994-2017 CC-BY 4.0 Instituto Geográfico Nacional, Madrid, en color en la versión electrónica). 


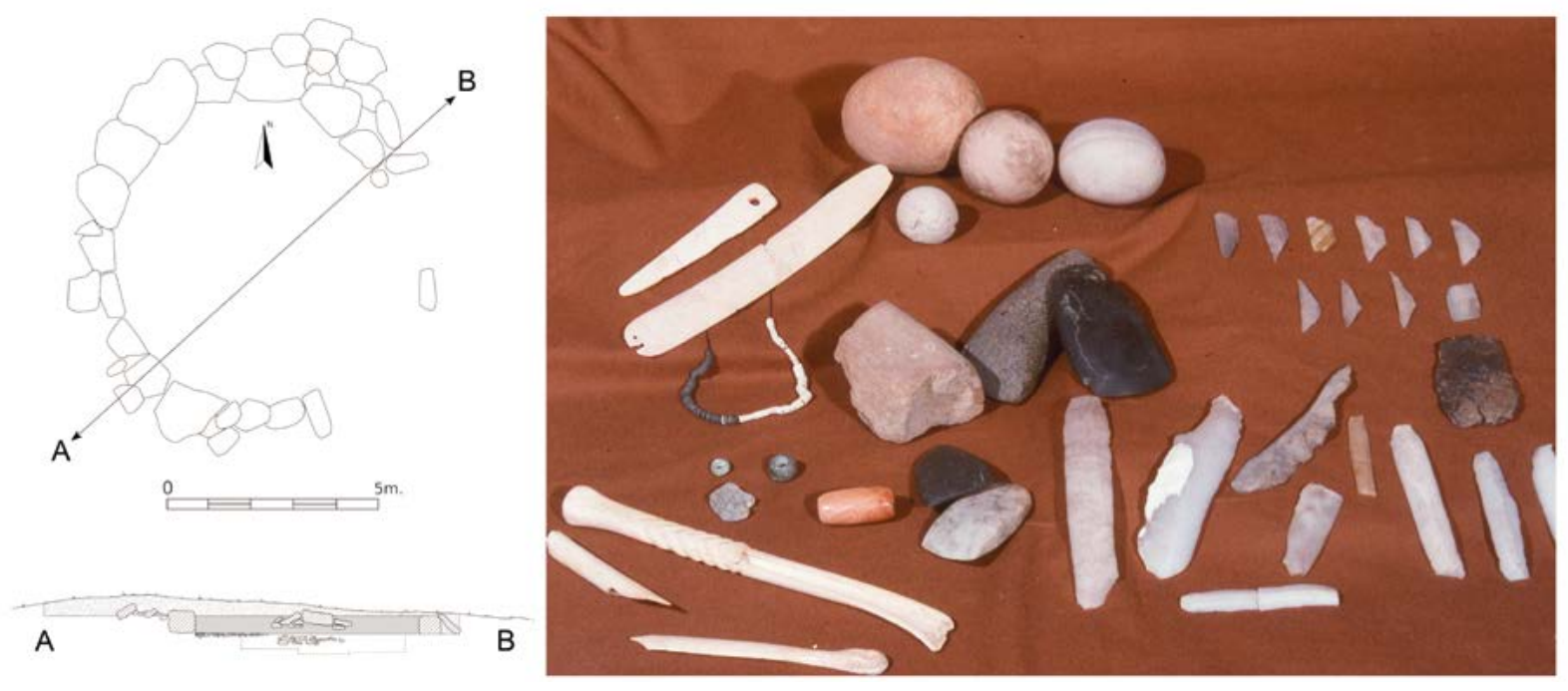

Fig. 2. Planta del dolmen de Los Zumacales (Simancas, Valladolid) y elementos del ajuar (imagen cedida por el Museo de Valladolid): útiles líticos pulimentados, grandes láminas y microlitos geométricos de sílex, punzones, colgantes e ídolos-espátula de hueso $(210 \times 15 \mathrm{~mm})$, cuentas exóticas de variscita y moscovitas verdes y caolinita roja, insignificante fragmento cerámico (en color en la versión electrónica).

tada en el túmulo de La Velilla, en Osorno (Palencia), donde consta que los alzados fueron de barro (Zapatero 1989). Además, ambos monumentos tienen en común que los grandes bloques de caliza delimitan una cámara sepulcral perfectamente circular. Por ello también se les denomina "redondiles". La presencia dentro de tales cámaras de osarios muy potentes y de ofrendas, similares a las de los sepulcros dolménicos de la península ibérica del IV milenio cal AC, completan el argumento a la hora de asimilar sin vacilaciones esta variedad de yacimientos al fenómeno megalítico.

El sepulcro de Los Zumacales se localiza a $1 \mathrm{~km}$ al este del pueblo de Simancas, en un punto de la terraza de $+740 \mathrm{~m}$ de la derecha del Pisuerga desde el que se ejerce un gran dominio visual. Ha sido objeto de dos campañas de excavación, en 1982 y 1989-1990. Cuenta con un túmulo redondo de $30 \mathrm{~m}$ de diámetro, muy mutilado, reforzado interiormente por varios peristalitos y una cámara central, también circular, de algo más de $5 \mathrm{~m}$, construida con lastras de calizas pontienses de los páramos aledaños. A la vista del alto número de bloques de este tipo diseminados por los alrededores del monumento, no se puede descartar que las paredes del redondil fueran también en su día de aparejo ciclópeo. Otro elemento estructural clave, por cuanto da pie a asignar al monumento la categoría de "sepulcro de corredor", es un pasillo, construido en este caso con verdaderos ortostatos, que se orienta al naciente solar y que, a tenor de lo poco conservado, tuvo una longitud mínima de $6 \mathrm{~m}$ por algo menos de 1 de anchura ${ }^{1}$ (Alonso Díez et al. 2015: 17-19).

En el interior de la cámara se descubrió un importante osario constituido por restos descoyuntados y en considerable desorden, salvo tres esqueletos en "perfecta conexión anatómica situados precisamente en las partes mejor conservadas del megalito, zonas norte y oeste. [...] con las extremidades ligeramente replegadas, sin presentar una disposición propiamente fetal" 2 . Según las primeras impresiones había unos veinte individuos entremezclados con dos centenares de piezas trabajadas sobre piedra, hueso y cerámica correspondientes al ajuar funerario. Entre ellas figuran 13 útiles líticos pulimentados (hachas/azuelas, esferoides y afiladeras) y 53 tallados (grandes láminas y microlitos geométricos de pedernal), punzones, colgantes e ídolos-espátula de hueso, 130 cuentas de collar, y unos insignificantes fragmentos de vasijas de barro (Fig. 2). Se han identificado de visu muchos de los pulimentados como grauwaca o silimanita, así como adornos de pizarra y conchas marinas (Alonso Díez et al. 2015: 25-28). Por arqueometría se ha certificado la presencia de cuentas de variscita y moscovitas verdes y caolinita roja (Villalobos 2015: cap. 3.2). Son materiales

\footnotetext{
1 Alonso Díez, M. 1985: El fenómeno megalítico en el Valle Medio del Duero: el sepulcro de corredor de Los Zumacales (Simancas). Memoria de licenciatura inédita. Valladolid. Universidad de Valladolid.

2 Véase n. 1, p. 79; Sampedro, C. 1990. Estudio antropológico de los restos procedentes del Sepulcro de Los Zumacales de Simancas (Valladolid). Laboratorio de Arqueozoología, Universidad Autónoma de Madrid, Madrid, inédito mecanografiado.
} 


\begin{tabular}{|c|c|c|c|c|c|}
\hline $\begin{array}{c}\text { Ref. } \\
\text { Laboratorio }\end{array}$ & Fecha BP & $\begin{array}{c}\text { Datación calibrada } \\
1 \sigma \text { cal AC }\end{array}$ & $\begin{array}{c}\text { Datación calibrada } \\
2 \sigma \text { cal AC }\end{array}$ & Muestra & Contexto \\
\hline GrN-17697 & $5310 \pm 90$ & $4253-4006$ & $4331-3973$ & Hueso de fauna & $\begin{array}{c}\text { Nivel } \\
\text { infratumular }\end{array}$ \\
\hline GrN-17693 & $4960 \pm 160$ & $3960-3543$ & $4224-3371$ & $\begin{array}{l}\text { Hueso humano (individuo } \\
\text { sin determinar) }\end{array}$ & Cámara \\
\hline GrN-17694 & $4950 \pm 160$ & $3956-3540$ & $4222-3368$ & $\begin{array}{l}\text { Hueso humano (individuo } \\
\text { sin determinar) }\end{array}$ & Cámara \\
\hline GrN-17695 & $4870 \pm 160$ & $3914-3382$ & $4041-3136$ & $\begin{array}{l}\text { Hueso humano (individuo } \\
\text { sin determinar) }\end{array}$ & Cámara \\
\hline
\end{tabular}

Tab. 1. Fechas BP y cal AC del sepulcro megalítico de Los Zumacales (Simancas, Valladolid) publicadas por Alonso Díez et al. (2015) con anterioridad al proyecto (PGC2018-099689-B-I00).

exóticos, lo que invita a ver en ellos "elementos de prestigio".

Las ofrendas de Los Zumacales constituyen un conjunto de materiales perfectamente representativos del horizonte megalítico inicial de la Meseta y denotan que el sepulcro ya estaba en uso a comienzos del IV milenio cal AC. Lo corroboran las tres fechas absolutas de huesos humanos ya publicadas (Tab. 1) - GrN 17693: $4960 \pm 160$ (4224-3371 cal AC 2 $)$ ); GrN 17694: $4950 \pm 160(4222-3368$ cal AC $2 \sigma)$ y

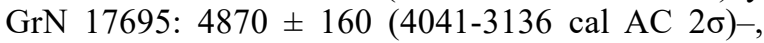
pese al problema de sus enormes desviaciones estándar. Una cuarta datación radiocarbónica, algo más precisa y referida en este caso a un nivel pretumular

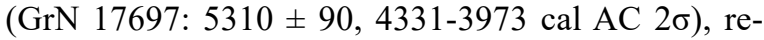
mite también a finales del $\mathrm{V}$ milenio cal $\mathrm{AC}$ con lo que la fecha de fundación de nuestro monumento parece relativamente bien fijada (Alonso Díez et al. 2015). Se trata de una fecha obtenida a partir de un hueso de fauna encontrado en un nivel de cenizas infratumular no muy profundo, con una extensión aproximada de $8 \mathrm{~m}^{2}$ y localizada entre el peristalito interior y el intermedio. Las cerámicas, huesos y restos de talla de sílex, encontradas en este pequeño depósito podrían sugerir que, en origen, fuera un basurero de una ocupación pretumular (Zapatero 1991; Alonso Díez et al. 1985). Este modesto testimonio de actividad previa a la construcción del monumento no es equiparable a evidencias de verdaderos espacios domésticos como los de La Velilla (Zapatero 1991) o Azután (Bueno 1991), pero facilita un verdadero término post quem para situar la construcción de los Zumacales entre finales del V y principios de IV milenio cal AC.

El desafío actual de este trabajo es determinar la duración de la trayectoria de esta tumba colectiva, algo que pasa inexcusablemente por el estudio del osario y por la datación sistemática de las inhumaciones que lo componen.

\section{LAS DATACIONES ABSOLUTAS DEL NMI DE LOS ZUMACALES}

\subsection{Estudio antropológico como punto de partida para la selección de muestras C14}

Los estudios cronológicos más recientes sobre series radiocarbónicas en conjuntos megalíticos de la península ibérica están proporcionando información muy interesante acerca de su funcionamiento. La temporalidad de las secuencias funerarias ha resultado sorprendentemente amplia, p. ej., en los dólmenes riojanos (Fernández Eraso y Mujika-Alustiza 2013) o en los monumentos megalíticos del sur peninsular (García Sanjuan et al. 2011; Aranda et al. 2017; Lozano y Aranda 2018). La mejor manera de establecer unos parámetros cronológicos claros a ese respecto es, por un lado, evitar la incertidumbre de las llamadas fechas "de vida larga" debido al conocido como "síndrome de la madera vieja" (Schiffer 1986), y, por otro, obtener dataciones directas de todos los individuos estudiados. La ventaja adicional es que ofrece datos cronológicos individualizados para cada una de las inhumaciones del monumento (Aranda et al. 2017: 262) ${ }^{3}$.

Un primer estudio, inédito, de los restos humanos depositados en la cámara del dolmen de Los Zumacales documentó un mínimo de 22 individuos, entre los cuales predominaba el grupo adulto sobre el subadulto y el masculino sobre el femenino, siendo muy reducido el número de infantiles ${ }^{4}$. Este tipo de composición poblacional, que ni difería demasiado del documentado en otros dólmenes meseteños, ni reflejaba en absoluto los esquemas de mortalidad propios de

\footnotetext{
${ }^{3}$ El procedimiento empleado en este tipo de análisis asume que los eventos datados son independientes y que tienen la misma probabilidad de ocurrir en cualquier momento de la escala temporal, es decir, se distribuyen uniformemente según el uniform phase model (Bronk Ramsey 2001: 357; Bronk Ramsey 2009a: 343).

4 Véase n. 1.
} 


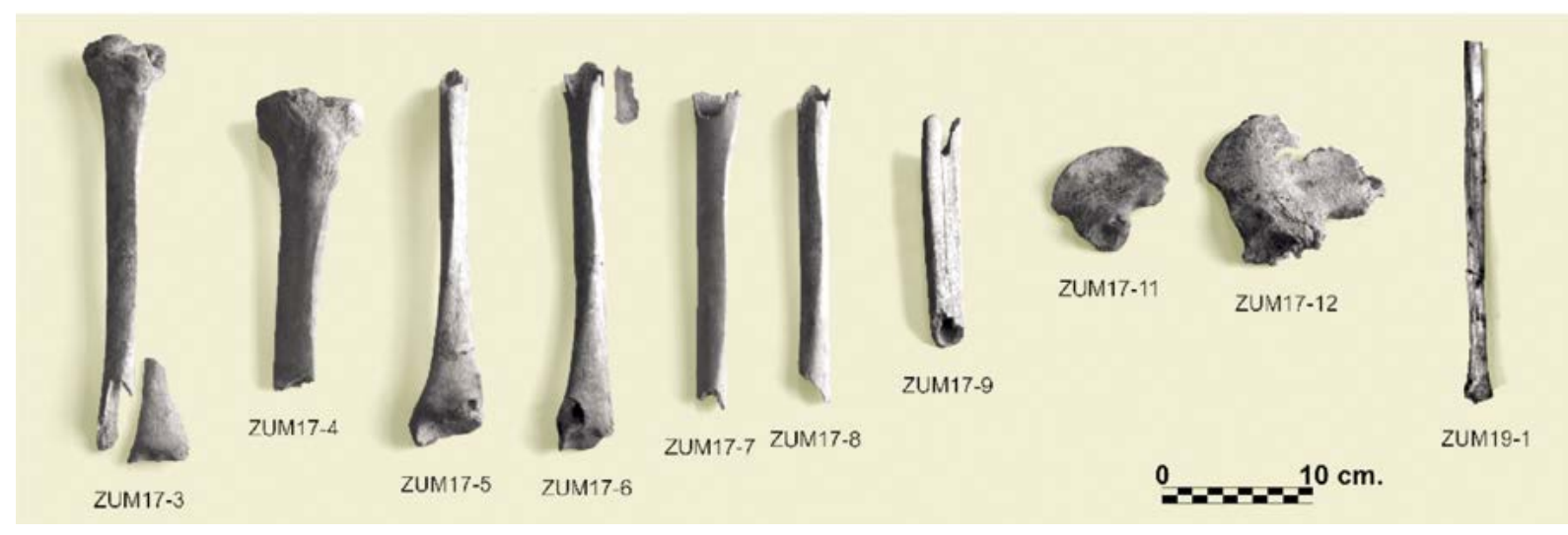

Fig. 3. Huesos seleccionados para la toma de muestras radiocarbónicas: tibias de individuos adultos, peroné de individuo subadulto y coxales de sujetos infantiles que conforman el NMI de la secuencia prehistórica de Los Zumacales (Simancas, Valladolid).

sociedades arcaicas preindustriales, daba pie a pensar en un "reclutamiento selectivo" de los individuos enterrados en los dólmenes (Masset 1986; Delibes 1995; Fernández-Crespo y de la Rúa 2015).

En una revisión posterior ${ }^{5}$, hemos establecido un NMI de 14. La identificación y recuento de los fragmentos óseos proporcionó un total de 1380 fragmentos de hueso aproximadamente. Esta cifra dista mucho de las 1800 piezas que se contabilizaron en el estudio previo donde, además, identifican "tres cráneos prácticamente completos que podrían corresponder a los únicos tres individuos que constan como recuperados, todavía en conexión anatómica, en la zona mejor conservada del monumento" ${ }^{\text {. }}$. Sin embargo, entre los fragmentos de cráneo solo se ha hallado un esplacnocráneo completo junto con un temporal y fragmentos de parietal, seguramente pertenecientes al mismo individuo. Cabe pensar que los cráneos "prácticamente" completos hayan sufrido una severa fragmentación, debido a su almacenamiento y transporte a lo largo del tiempo, o bien que falten piezas en la colección, dada la diferencia indicada en los totales de fragmentos.

El número mínimo de individuos adultos se determinó mediante el recuento de tibias izquierdas identificadas por la epífisis distal y el agujero nutricio proximal. El grupo de edad subadulto constaba de un mínimo de 5 sujetos, resultante de cotejar los huesos largos y coxales con el grado de desarrollo y erupción dental. Uno de ellos, un peroné (Fig. 3, muestra ZUM19.1), se ha conseguido individualizar parcialmente gracias a que los fragmentos presentaban huellas patológicas idénticas y un proceso de fusión de epífi-

\footnotetext{
5 Santa Cruz del Barrio, A. 2016: Análisis antropológico de los restos óseos del sepulcro megalítico de Los Zumacales (Simancas, Valladolid). Trabajo de Fin de Máster inédito. Universidad de Granada.

${ }^{6}$ Véase n. 1, p. 2.
}

sis compatible con un sujeto entre los 12 y 19 años. La composición poblacional de este segundo estudio antropológico (Tab. 2) apunta igualmente a un predominio de individuos adultos masculinos sobre otros grupos, aunque en proporciones menos significativas.

La metodología utilizada en la estimación de edad de la muestra se ha basado, sobre todo en el esqueleto postcraneal dada la considerable fragmentación de los restos craneales. Se han podido rescatar escasos maxilares, mandíbulas y casi una cincuentena de dientes.

La edad en individuos adultos se ha determinado, primero, por su grado de desgaste dental (Brothwell 1981; Lovejoy 1985); después por los fragmentos de sínfisis púbica del hueso coxal, según la revisión de Todd (1920) por Meindl et al. (1985). Por último, se ha considerado la superficie auricular de la articulación sacroilíaca (Lovejoy et al 1985).

Los fragmentos de hueso correspondientes a individuos inmaduros, maxilares y mandíbulas han han permitido estimar el intervalo de edad más específico

\begin{tabular}{|c|c|c|c|}
\hline Grupos de edad & $\begin{array}{l}\text { Rangos } \\
\text { de edad }\end{array}$ & Individuos & Porcentaje \\
\hline Neonatos & 0 & 0 & \multirow{4}{*}{$35,71 \%$} \\
\hline Infantil I & $0-3$ & 1 & \\
\hline Infantil II & $3-12$ & 2 & \\
\hline Adolescente & $12-20$ & 2 & \\
\hline Adulto joven & $20-35$ & 3 & \multirow{3}{*}{$64,28 \%$} \\
\hline Adulto & $35-50$ & 2 & \\
\hline $\begin{array}{l}\text { Adulto de edad } \\
\text { indeterminada }\end{array}$ & ¿? & 4 & \\
\hline Total & & 14 & $100 \%$ \\
\hline
\end{tabular}

Tab. 2. Distribución por grupos de edad del número mínimo de individuos del dolmen de Los Zumacales (Simancas, Valladolid). 
y fiable a partir del desarrollo dental (AlQahtani et al. 2010). La estimación de edad en subadultos mediante el esqueleto postcraneal se ha basado principalmente en el desarrollo y fusión de las epífisis de huesos largos (Brothwell 1981; Scheuer y Black 2000).

La presencia de procesos artrósicos en vértebras y articulaciones como indicador aproximativo de edades más avanzadas ha sido escasamente detectada en la muestra. No obstante, hay más factores causantes y favorecedores de estos procesos (sexo, nutrición, genética, estrés biomecánico, etc.) (Jimenez-Brobeil 2012). La actividad física intensifica y adelanta la edad de aparición de signos de artrosis. Además, este factor aparece como principal causante de la artrosis vertebral en poblaciones prehistóricas, lo cual adelanta su edad de aparición (Jiménez-Brobeil et al. 2010).

El diagnóstico sexual solo se ha podido aplicar en algunos fragmentos del coxal o del cráneo. La estimación del sexo a partir del cráneo se ha reducido a observar la apófisis mastoides y la morfología del reborde supraorbitario en casos aislados, así como la eminencia mentoniana y el ángulo goniaco en mandíbulas (Ferembach et al. 1980; Buikstra y Ubelaker 1994). Estos rasgos morfológicos no son demasiado evidentes por la fragmentación de los huesos. Además, en especial la apófisis mastoides se considera en Antropología física poco fiable como marcador sexual si se desconoce la caracterización craneal de la población y si no se coteja con otros elementos óseos (White et al. 2012).

Los fragmentos de coxal han sido los marcadores morfológicos para la discriminación de sexo en el esqueleto postcraneal (Buikstra y Ubelaker 1994). Se desaconseja la diferenciación sexual a partir de restos aislados de individuos inmaduros dado que los rasgos morfológicos sexuales se definen una vez pasada la pubertad (Scheuer y Black 2000).

En definitiva, la estimación de sexo basada en los criterios citados tan solo ha permitido establecer un mínimo de 3 individuos varones, 2 mujeres y un alofiso. Es una representación bastante poco significativa de la probable composición originaria de la población del sepulcro simanquino.

\subsection{Selección de muestras}

La selección ha tenido en cuenta los resultados del reciente estudio antropológico ${ }^{7}$ y la documentación de las excavaciones arqueológicas de M. Alonso Díez ${ }^{8}$. En ella se asegura que todos los huesos humanos pro-

\footnotetext{
7 Véase n. 5.

8 Véase n. 1.
}

cedían de la cámara, motivo por el cual se han atribuido a una misma unidad estratigráfica. Durante su recuperación los huesos se guardaron en bolsas individualizadas y con el registro espacial correspondiente. Sin embargo, se ha desestimado esta información por el carácter revuelto del osario ${ }^{9}$. Además, por desgracia el conjunto esquelético en posición anatómica que se mencionaba y podría coincidir con las últimas deposiciones funerarias, no ha sido identificado durante la revisión antropológica.

Una prueba adicional del carácter removido del osario es que falta, en general, un estricto orden decreciente de antigüedad en las dataciones prehistóricas conforme disminuye la profundidad de sus correspondientes muestras. Las fechas más modernas sí tienden a ser las de individuos o huesos aparecidos en cotas altas (Tab. 3). Sin embargo, no hay evidencias arqueológicas claras de intrusiones, más allá de "algunos destrozos" contemporáneos producidos por las tareas de roturación. Por todo ello, en coincidencia con lo planteado hasta ahora (Alonso Díez et al. 2015: 16 y 30 ), lo lógico es considerar una sola etapa o fase de actividad funeraria, no muy extensa e ininterrumpida.

Por último, debido a la desmineralización y mala conservación de los huesos infantiles, así como la costosa identificación de sus intervalos de edad, trabajamos prudentemente con 12 muestras del total de 14 individuos atestiguados, de los que 10 corresponderían a cronología prehistórica. Como indicamos, aquí se analizan las fechas correspondientes a la etapa prehistórica del dolmen. Además dos de los nueve adultos datados tienen fechas de época medieval (Poz-93608 (ZUM17-1): $1060 \pm 30$ BP; Poz-93609 (ZUM17-2): $1175 \pm 30 \mathrm{BP}$ ) muy próximas entre sí y cercanas al año 1000 cal AD. Destacamos este hecho completamente inesperado ya que ningún elemento material permitía sospechar intrusiones de época histórica.

\subsection{Resultados}

Las muestras fueron procesadas en el Poznam Radiocarbon Laboratory, en colaboración con el AMS Laboratory de la A. Mickiewicz University (Polonia). Las fechas obtenidas (Tab. 3) han sido calibradas con el programa OxCal versión 4.3.2 (Bronk Ramsey 2009a) de acuerdo con la curva atmosférica IntCal13 (Reimer et al. 2013). La primera impresión que ofrecen es la de una secuencia agrupada, bastante bien definida en sus momentos más antiguos. La salvedad son un par de fechas -sobre todo Poz-93612 (4410 \pm 35 BP), cuya calibración se sitúa entre 3093 y 2936

9 Véase n. 1.

Trab. Prehist., 77, N. ${ }^{\circ}$ 1, enero-junio 2020, pp. 130-147, ISSN: 0082-5638 https://doi.org/10.3989/tp.2020.12250 
cal AC $(1 \sigma)$ y entre 3321 y 2915 cal AC $(2 \sigma)-$ que podrían prolongar casi mil años el uso funerario del dolmen. No obstante, asumir la hipótesis de un uso tan dilatado a partir de una única fecha discordante parece arriesgado, por lo que se ha recurrido al tratamiento estadístico bayesiano. Su fin es reajustar los intervalos de las fechas calendáricas que ofrece la secuencia radiocarbónica (Bronk Ramsey 1995). Se evitan así interpretaciones erróneas deducidas de la mera observación de las fechas calibradas, explicables amplio rango de dispersión estadística que presenta cada datación (Bayliss et al. 2007).

La construcción de modelos bayesianos permite realizar estimaciones interpretativas (posterior information) acerca de las fases o secuencias de una actividad arqueológica, combinando información previa (prior information) con las probabilidades estandarizadas proporcionadas por las fechas de radiocarbono (Bronk Ramsey 1995). Las fechas se han analizado con el programa OxCal (v.4.2.3.) para el tratamiento bayesiano usando las funciones Boundary Start, Boundary End, Span y Outlier Model (Bronk Ramsey 2009b). En la interpretación, se han tenido en cuenta los valores del índice de consistencia general del modelo, que ha de ser mayor de $60 \%$ para aceptar su coherencia interna (Bronk Ramsey 1995). Además se ha prestado especial atención a los valores del índice de consistencia individual de cada una de las fechas, en especial de las re-

\begin{tabular}{|c|c|c|c|c|c|c|c|}
\hline Muestra & $\begin{array}{c}\text { Ref. } \\
\text { Laboratorio }\end{array}$ & Fecha BP & \begin{tabular}{|c|} 
Datación calibrada \\
$1 \sigma$ cal AC
\end{tabular} & \begin{tabular}{|c} 
Datación calibrada \\
$2 \sigma$ cal AC
\end{tabular} & Colágeno & Muestra & Contexto \\
\hline ZUM17-6 & Poz-93536 & $5020 \pm 40$ & $3936-3715$ & $3944-3709$ & $\begin{array}{l}1,4 \% \mathrm{~N} \\
8,2 \% \mathrm{C} \\
5 \% \text { coll } \\
\end{array}$ & $\begin{array}{l}\text { Tibia humana } \\
\text { de individuo } \\
\text { adulto }\end{array}$ & $Z=-82$ \\
\hline ZUM17-11 & Poz-93542 & $5010 \pm 40$ & $3923-3712$ & $3944-3704$ & $\begin{array}{c}2,2 \% \mathrm{~N} \\
9,8 \% \mathrm{C} \\
7,3 \% \text { coll }\end{array}$ & $\begin{array}{c}\text { Coxal } \\
\text { humano de } \\
\text { individuo } \\
\text { subadulto }\end{array}$ & $Z=-87$ \\
\hline ZUM17-7 & Poz-93537 & $4990 \pm 40$ & $3894-3706$ & $3941-3661$ & $\begin{array}{c}1,9 \% \mathrm{~N} \\
6,7 \% \mathrm{C} \\
4,4 \% \mathrm{coll}\end{array}$ & $\begin{array}{l}\text { Tibia humana } \\
\text { de individuo } \\
\text { adulto }\end{array}$ & $Z=-68$ \\
\hline ZUM17-12 & Poz-93543 & $4990 \pm 35$ & $3797-3709$ & $3938-3664$ & $\begin{array}{l}2,5 \% \mathrm{~N} \\
9,4 \% \mathrm{C} \\
8 \% \text { coll }\end{array}$ & $\begin{array}{c}\text { Fémur } \\
\text { humano de } \\
\text { individuo } \\
\text { subadulto }\end{array}$ & $Z=-60-75$ \\
\hline ZUM17-8 & Poz-93538 & $4990 \pm 35$ & $3797-3709$ & $3938-3664$ & $\begin{array}{c}2 \% \mathrm{~N} \\
7,3 \% \mathrm{C} \\
5,6 \% \text { coll }\end{array}$ & $\begin{array}{l}\text { Tibia humana } \\
\text { de individuo } \\
\text { adulto }\end{array}$ & $Z=-70$ \\
\hline ZUM17-4 & Poz-93611 & $4975 \pm 35$ & $3787-3706$ & $3924-3658$ & $\begin{array}{c}3,3 \% \mathrm{~N} \\
10,6 \% \mathrm{C} \\
7,3 \% \text { coll }\end{array}$ & $\begin{array}{l}\text { Tibia humana } \\
\text { de individuo } \\
\text { adulto }\end{array}$ & $Z=-82$ \\
\hline ZUM17-3 & Poz-93610 & $4895 \pm 35$ & $3697-3649$ & $3762-3637$ & $\begin{array}{c}0,9 \% \mathrm{~N} \\
9,1 \% \mathrm{C} \\
2,8 \% \text { coll }\end{array}$ & $\begin{array}{l}\text { Tibia humana } \\
\text { de individuo } \\
\text { adulto }\end{array}$ & $Z=-116$ \\
\hline ZUM19-1 & Poz-112689 & $4820 \pm 35$ & $3650-3536$ & $3692-3522$ & $\begin{array}{c}3,2 \% \mathrm{~N} \\
11,6 \% \mathrm{C} \\
7,1 \% \mathrm{coll}\end{array}$ & $\begin{array}{c}\text { Peroné } \\
\text { humano de } \\
\text { individuo } \\
\text { subadultos }\end{array}$ & $Z=-60-75$ \\
\hline ZUM17-9 & Poz-93539 & $4680 \pm 40$ & $3518-3374$ & $3628-3366$ & $\begin{array}{l}2,2 \% \mathrm{~N} \\
7,7 \% \mathrm{C} \\
4 \% \text { coll }\end{array}$ & $\begin{array}{l}\text { Tibia humana } \\
\text { de individuo } \\
\text { adulto }\end{array}$ & $Z=-62$ \\
\hline ZUM17-5 & Poz-93612 & $4410 \pm 35$ & 3093-2936 & $3321-2915$ & $\begin{array}{c}3,9 \% \mathrm{~N} \\
10,9 \% \mathrm{C} \\
8,8 \% \text { coll }\end{array}$ & $\begin{array}{l}\text { Tibia humana } \\
\text { de individuo } \\
\text { adulto }\end{array}$ & $Z=-43$ \\
\hline
\end{tabular}

Tab. 3. Serie radiocarbónica partir de muestras de 10 individuos del dolmen de Los Zumacales (Simancas, Valladolid) (fechas BP y AC calibradas a $1 \sigma$ y $2 \sigma$ ). El valor de "z" se corresponde con la profundidad en relación con "la parte más elevada del terreno" (Alonso Díez 1985: 11). N nitrógeno, C carbono, coll colágeno. 
gistradas en las cotas más altas y que podían corresponderse con las últimas deposiciones.

$\mathrm{Al}$ no haberse registrado más que un nivel arqueológico, la actividad funeraria del dolmen se interpreta a priori como una única secuencia cultural. Ello justifica que para el modelado bayesiano hayamos asignado todas las fechas a una sola fase. Como ya se mencionó, esta interpretación se debe al carácter colectivo de los enterramientos dolménicos y a la consideración como un conjunto único de todo el osario de la cámara de Los Zumacales, sin espacios divididos o elementos de ajuar atribuibles a épocas diferentes ${ }^{10}$. Este hecho nos priva de cualquier referencia secuencial que $a$ priori pudiera ser de interés para la construcción del modelo estadístico. Otra razón de peso para descartar la identificación de niveles estratigráficos claros es la alteración de los osarios dolménicos.

Además, en contraste con los fenómenos de reutilización, o al menos de intrusión, que manifiestan numerosos dólmenes, Los Zumacales tiene un ajuar homogéneo, propio de un momento megalítico inicial sin, p. ej., puntas de flecha de retoque plano, elementos de ajuar campaniformes u otros materiales posteriores. Este detalle llevó a plantear muy sugestivamente un uso del espacio funerario más bien ceñido a una de las primeras fases dolménicas en la Submeseta Norte, caracterizada por ofrendas como los microlitos geométricos y las espátulas tipo San Martín-El Miradero $^{11}$ (Alonso Díez et al. 2015: 30). De este modo, el modelado bayesiano del nuevo conjunto de dataciones, correspondientes a un porcentaje considerable de los individuos inhumados, se erige en una herramienta prometedora tanto para confirmar la hipótesis de un uso funerario asimilable a una breve secuencia cultural, como para contextualizar de modo más preciso el inicio y final de dicho uso.

\section{DISCUSIÓN: EL COMPORTAMIENTO CRONOLÓGICO DE LA UTILIZACIÓN FUNERARIA DE LOS ZUMACALES}

\subsection{Primer modelo de combinación estadística}

Este modelo, con un índice de consistencia válido $\left(\mathrm{A}_{\text {model }}: 82 \%\right)$, considera que todos los individuos prehistóricos datados $(\mathrm{n}=10)$ podrían pertenecer a una misma fase prolongada y continuada en el tiempo (Fig. 4). De acuerdo con él, la duración de la secuencia funeraria podría ser de 459-646 años $(1 \sigma)$ y 434-877 años $(2 \sigma)$ (Fig. 5), lo cual no concuerda con la hipótesis de clau-

\footnotetext{
10 Véase n. 1.

11 Véase n. 1, p. 79.
}

sura temprana formulada para este dolmen. Es más, si se consideran los límites de esta secuencia, con un inicio probable entre 3962-3788 cal AC y un final entre 3321-3153 cal AC (con un 68,2\% de probabilidad), se entendería que el sepulcro de Los Zumacales aún seguiría abierto durante el último tercio del IV milenio cal AC. En suma, este modelo plantearía la posibilidad de una considerable prolongación de la actividad funeraria, contemporánea en sus momentos finales de otros dólmenes como el palentino de La Velilla (Zapatero 2012), el burgalés de Las Arnillas (GrN-12124: $4575 \pm$ 40 BP) (Delibes et al. 1987), o las fases medias y finales de monumentos riojanos como Alto de la Huesera, Chabola de La Hechicera o El Sotillo (Fernández-Eraso y Mújika 2013). En todos los citados -no en Los Zumacales- otros materiales como las puntas de flecha de retoque plano anticipaban un funcionamiento tardío en fechas próximas al $3000 \mathrm{cal}$ AC.

Este primer modelo, aunque estadísticamente sólido, encajaría dentro de la clásica interpretación de los dólmenes como "tumbas para la eternidad" (Delibes 2000: 301; Delibes y Rojo 2002: 29). Este largo uso diacrónico y apertura recurrente (Delibes 1995, 2010) últimamente se cuestiona gracias a las nuevas series de dataciones dolménicas que proponen algunas secuencias de uso más bien cortas (Fernández-Eraso y Mújika 2013; Aranda y Lozano 2014; Aranda et al. 2017; Fernández-Crespo et al. 2018), cuando no clausuras inmediatas tras el uso inicial, como se defiende a propósito de las tumbas-calero del Valle de Ambrona (Rojo Guerra et al. 2005).

En el dolmen de Los Zumacales, las evidencias arqueológicas y el bajo NMI establecido obligan a prestar atención al bajo índice de consistencia individual de algunas dataciones. Sucede con Poz-93612 $(4410 \pm 35 \mathrm{BP})$, la más moderna del conjunto, que en el modelo general de fase única es estadísticamente inconsistente $\left(\mathrm{A}_{\text {individual }}=42 \%\right)$ (Fig. 6). Podría ser considerada como un valor atípico (outlier) dentro del modelo general de fase única, bien por errores de medición, de contaminación o de anomalías en el reservorio isotópico ${ }^{12}$, bien por no pertenecer realmente al modelo temporal que se propone (Bronk Ramsey 2009b). Sin embargo, rechazar esta fecha del modelo y prescindir de ella por atípica llevaría también a cuestionar otra, Poz-93539 (4680 \pm 40$)$, con un índice de consistencia individual bajo (A=58 \%) (Fig. 7). Ambas dataciones corresponden a los individuos registrados en las cotas más altas del yacimiento, por cuanto no habría que descartar su segregación estratigráficotemporal respecto a los restantes individuos.

\footnotetext{
12 Las mediciones radiométricas podrían alterarse en función los valores de $\delta 13 \mathrm{C}$ y $\delta 15 \mathrm{~N}$ en colágeno presente en las muestras, cuyo análisis actualmente se encuentra en proceso.
} 


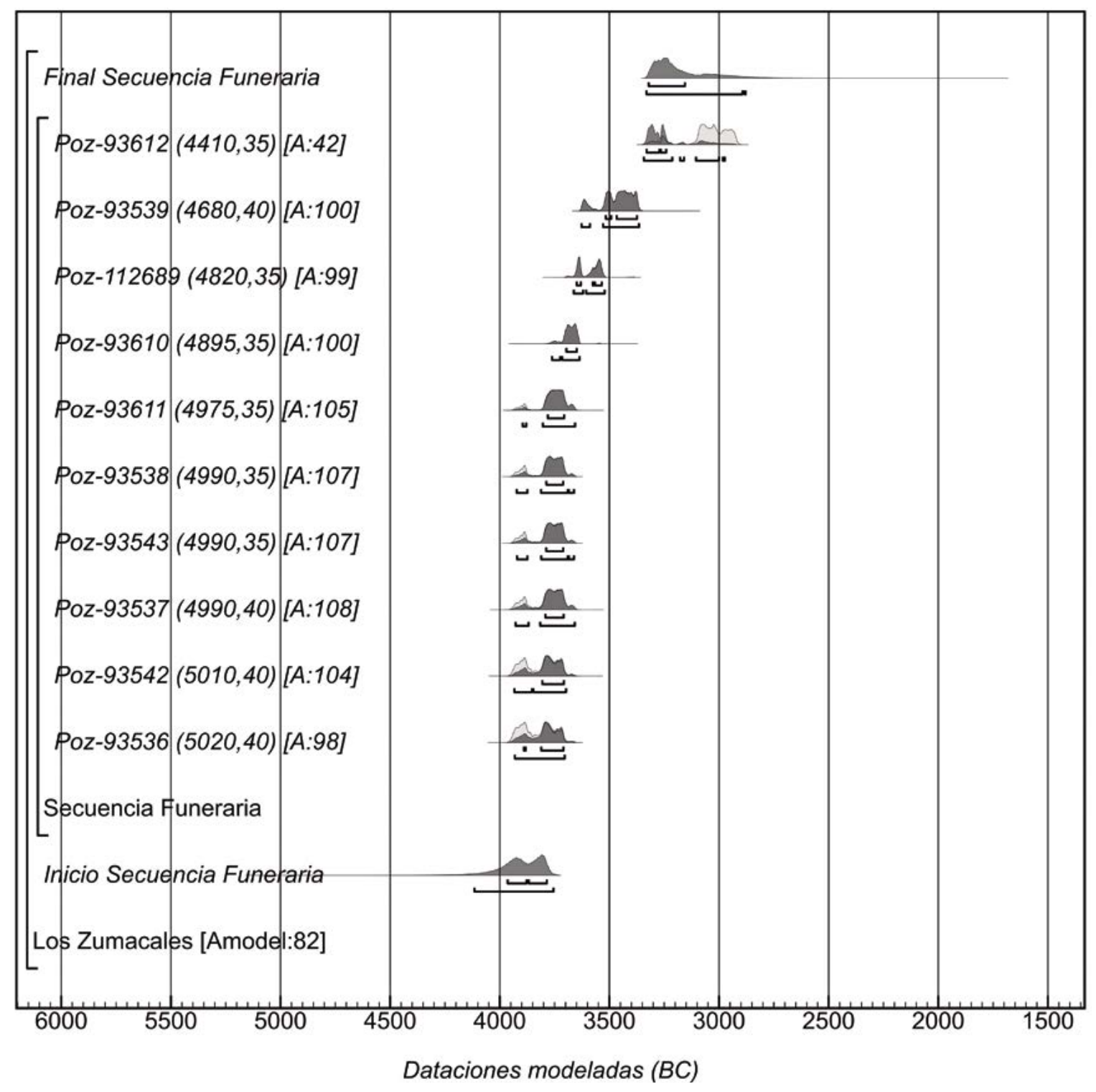

Fig. 4. Primer modelo bayesiano (Uniform phase model) de la secuencia prehistórica completa del dolmen de Los Zumacales (Simancas, Valladolid). Las muestras datadas son huesos humanos.

\subsection{Segundo modelo de combinación estadística}

El tratamiento bayesiano en el programa de software OxCal v.4.3.2 permite analizar estos valores atípicos con un modelo específico que ha sido el recomendado por Bronk Ramsey (2009b) para muestras fuera de contexto discordantes con el intervalo temporal esperado. El análisis se realiza mediante la función Outlier Model ("General", T(5), U(0,4), "t"), con una probabilidad del $5 \%$ para los outliers. En nuestro caso, si bien el modelo resultante es consistente $\left(\mathrm{A}_{\text {overall }}=\right.$
$102,6 \%$ y $_{\text {model }}=104 \%$ ), la fecha Poz-93612 (4410 $\pm 35 \mathrm{BP}$ ) presenta un índice de consistencia individual igualmente bajo $\left(\mathrm{A}_{\text {individual }}=38 \%\right.$, O: $26 \%$ de probabilidad a posteriori) por lo que podría responder a un valor atípico y por tanto excluible del modelo (Fig. 6). Al prescindir de esa fecha, el índice de consistencia individual de la inmediatamente posterior (Poz-93539, $4680 \pm 40)$ también sería cuestionable $(\mathrm{A}=58 \%)$, aunque al no mostrarse como un valor atípico claro, se incluirá en el análisis (Fig. 7). Todo lo contrario, sucede con las siete primeras fechas analizadas, con 


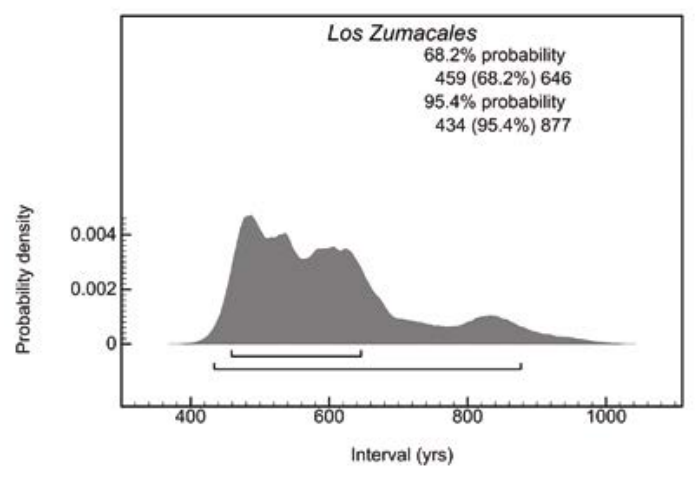

Fig. 5. Probabilidades de la duración total de la secuencia funeraria del dolmen de Los Zumacales (Simancas, Valladolid). índices siempre coherentes dentro de este análisis, por lo que el conjunto es más probable que pertenezca a la misma fase temporal.

Según este segundo modelo, siempre que se excluya la fecha Poz-93612 $(4410 \pm 35 \mathrm{BP})$ por la alta probabilidad de ser un valor atípico, la duración probable de la secuencia funeraria del dolmen de Los Zumacales sería más breve, entre 120 y 229 años $(1 \sigma)$

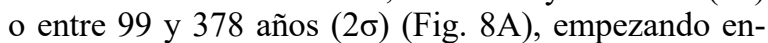
tre 3846 y 3756 y finalizando entre 3627 y $3550 \mathrm{cal}$ $\mathrm{AC}(1 \sigma)$, con mejor índice este segundo modelo $\left(\mathrm{A}_{\text {overall }}=98 \%\right)$. Si se prescinde también de las fechas situadas a mediados del IV milenio cal AC (Poz-93539, $4680 \pm 40$; Poz-112689, $4820 \pm 35$ ), el programa permite realizar la combinación estadística del resto de
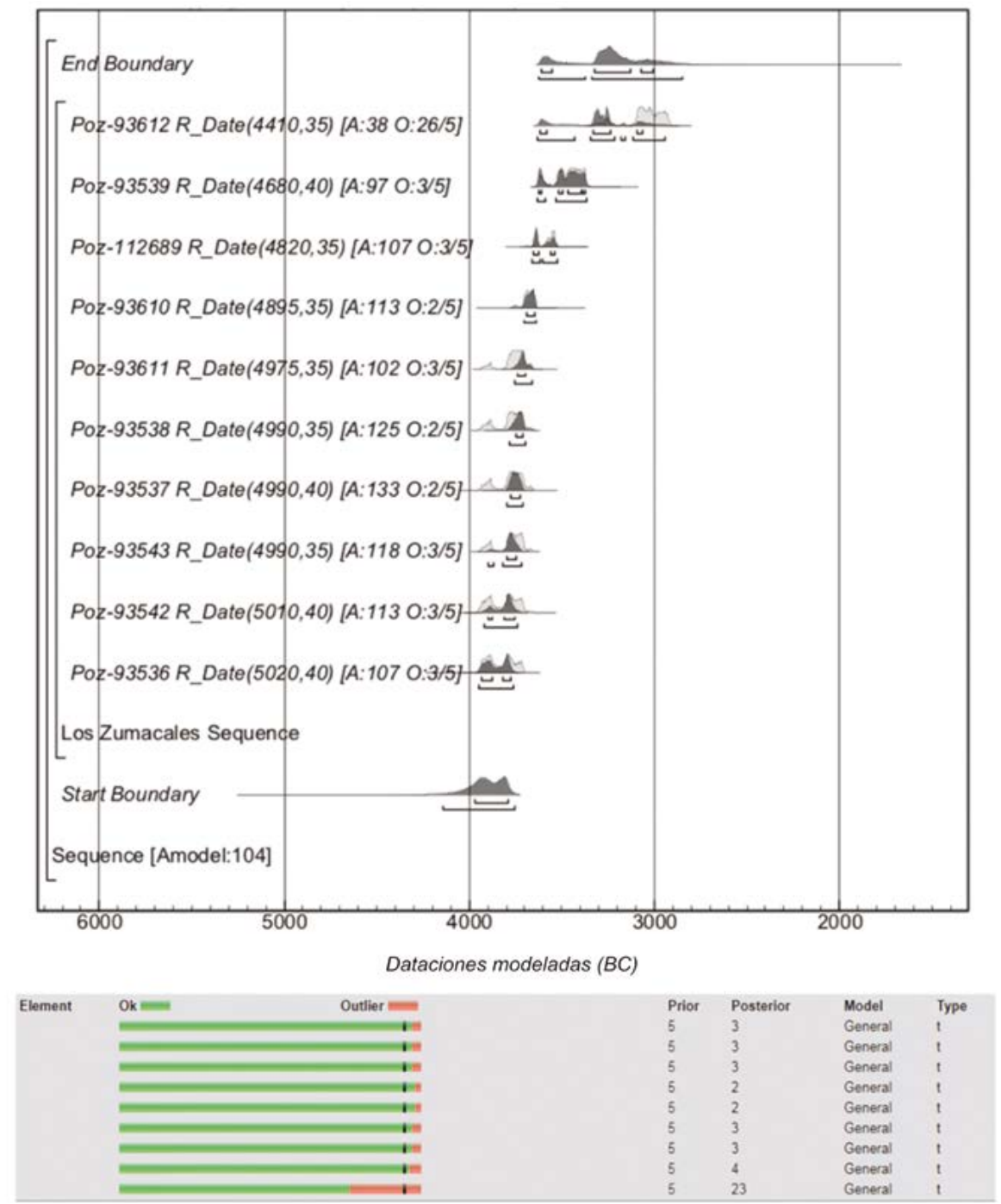

Fig. 6. Segundo modelo bayesiano (Outlier Model) de la secuencia radiocarbónica de Los Zumacales (Simancas, Valladolid).

Trab. Prehist., 77, N. ${ }^{\text {1 }}$, enero-junio 2020, pp. 130-147, ISSN: 0082-5638 


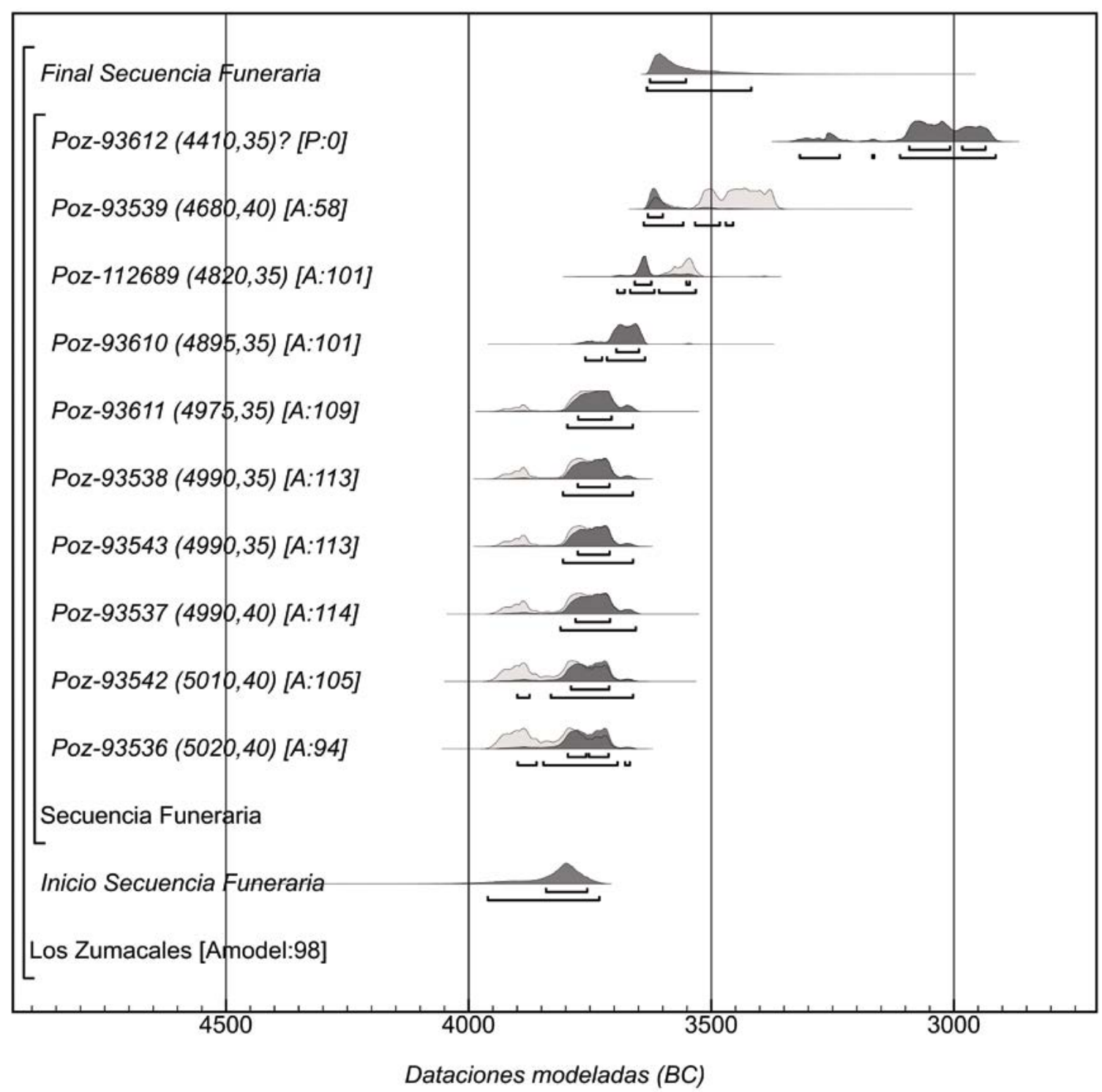

Fig. 7. Modelo bayesiano definitivo (Uniform phase model) de la secuencia prehistórica completa del dolmen de Los Zumacales (Simancas, Valladolid), excluyendo la fecha atípica (outlier) Poz-93612 (4410 \pm 35 ).

dataciones con la prueba de contemporaneidad (Ward y Wilson 1978) en la función R_Combine del programa OxCal $[\mathrm{T}=7,6 ;(5 \% 12,6)]$. El resultado apoya la hipótesis de un primer y principal uso funerario, considerablemente breve, del dolmen, coincidente con los inicios del megalitismo regional. Dicha función ${ }^{13} \mathrm{com}-$ prueba la consistencia interna de las fechas mediante una prueba $\chi^{2}$, por lo que lo más probable es que se

${ }^{13}$ University of Oxford. Radiocarbon Accelerator Unit OxCal Program v3.10 (c) Copyright Christopher Bronk Ramsey 2005 (https://c14. arch.ox.ac.uk/oxcal3/arch_cmb.htm). trate de un depósito rápido de los siete primeros individuos datados.

De todo ello parece deducible que, en efecto, la mayoría de los individuos fueron enterrados durante un intervalo de tiempo bastante reducido (entre $0 \mathrm{y}$ 130 años con un 95,4\% de probabilidad) simultáneamente o a lo largo de cinco generaciones, asumiendo una media de 25 años por cohorte generacional (Fig. 8B). Podría también intuirse una fase de enterramiento ligeramente posterior, sin embargo, como no hay razones estadísticas de peso para confirmar la existencia de un hiato temporal entre ambas secuen- 
cias, podríamos atribuir el descenso de la frecuencia de enterramientos a un descenso de la actividad funeraria hacia la mitad del IV milenio cal AC.

Una última consideración sobre la temporalidad del dolmen de Los Zumacales atañe al momento de su contrucción. A ese respecto solo se dispone de la fecha procedente del contexto infratumular realizada sobre hueso de fauna (GrN-17697, $5310 \pm 90)$, que es un buen término post quem para la construcción del monumento. En cambio, por si sola, no basta para establecer una fase previa a la elevación del túmulo funerario, y distorsiona demasiado sus límites temporales. Pese a la escasa información disponible, el modelo bayesiano presenta un índice de consistencia aceptable $(\mathrm{A}=98 \%$ ). Ello permite ubicar la construcción de Los Zumacales entre el 4137-3886 cal AC $(1 \sigma)$ o 4247 3797 cal AC $(2 \sigma)$, momento probable del final de la fase infratumular. La secuencia funeraria se iniciaría en el intervalo 3854-3761 cal AC con un 68,2\% de probabilidad (Fig. 9).

La consecuencia inmediata de analizar la trayectoria funeraria del dolmen de Los Zumacales a partir de la datación de cada individuo analizado es la ligera modificación de los datos demográficos de las secuencias sepulcrales. Se asume que ninguna de las muestras fechadas concierne al conjunto de enterramientos, cuya representatividad está afectada por los problemas de conservación de las muestras dolménicas (Robb 2016). Sin embargo, ello no impide que la interpretación cronológica propuesta sea representativa de la actividad funeraria en el dolmen, dado que las diez dataciones prehistóricas, seleccionadas al azar, abarcan una buena parte de ellos.

Otras precisiones necesarias sobre los datos demográficos (NMI=14, de los que se dataron 12) se rela- cionan con la citada presencia de dos individuos adultos con cronologías medievales. La falta de testimonios de nuevos usos funerarios en época histórica haría sospechar de la validez de las dataciones, pero la proximidad temporal y la coherencia entre sus valores (entre los siglos IX y X cal DC) justifican su atribución a dos sujetos medievales. Prescindiendo de ellos, los valores absolutos de la composición poblacional en la secuencia funeraria prehistórica datada son un $60 \%$ de adultos frente a un $40 \%$ de subadultos aproximadamente, dos de los cuales son individuos infantiles enterrados en los momentos más antiguos.

Se trata de una muestra poblacional demasiado pequeña para conocer con precisión los patrones de mortalidad propios de la comunidad titular de este sepulcro y, en consecuencia, para un análisis fiable de los parámetros demográficos de este yacimiento (BocquetAppel y de Miguel 2002). Ello no impide reconocer la evidente infrarrepresentación de individuos infantiles tan característica en los monumentos megalíticos meseteños (Delibes 1995). Sin embargo, la ligera alteración de los datos poblacionales resultante del análisis de la temporalidad de las secuencias funerarias demuestra los sesgos en la representación de grupos de edad y sexo debidos al carácter acumulativo de las tumbas colectivas. Por ello la variable cronológica debería tenerse muy en cuenta al interpretar la composición poblacional de este tipo de sepulcros, especialmente si no hay registro o constancia de diferentes niveles de ocupación. Se requieren buenas cronologías funerarias para interpretar y comprender la dimensión temporal y social de los sepulcros colectivos prehistóricos.

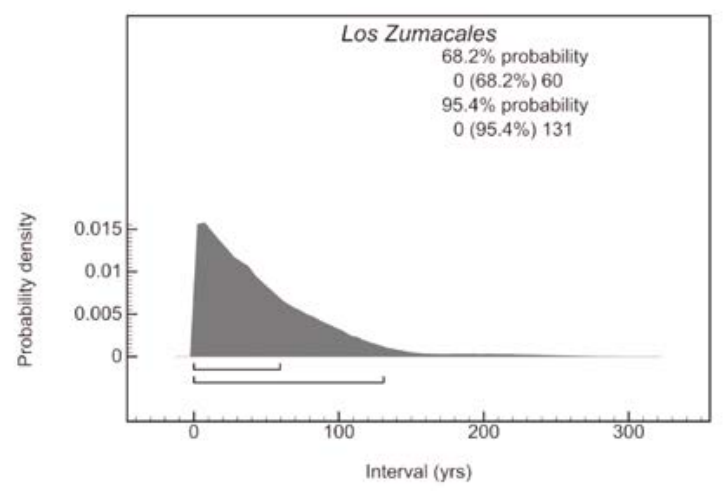

Fig. 8. Probabilidades de duración de la secuencia funeraria del dolmen de Los Zumacales (Simancas, Valladolid) a partir de la serie radiocarbónica: A. según el segundo modelo (Outlier model); B. mediante la combinación de fechas que pasan la prueba de contemporaneidad (función R_Combine). Dicha combinación sugiere un primer uso funerario más breve. 


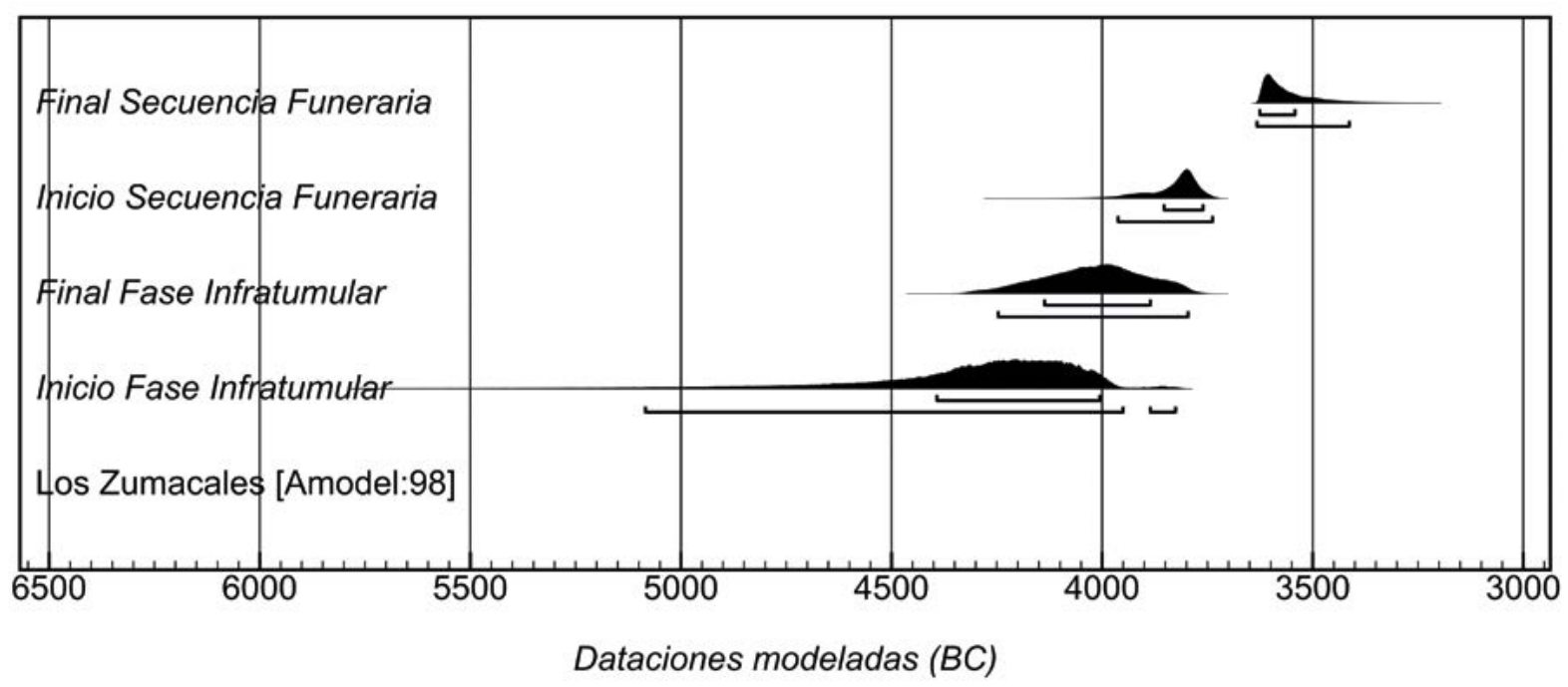

Fig. 9. Modelo bayesiano del dolmen de Los Zumacales (Simancas, Valladolid) incluyendo la información infratumular (GrN $17697: 5310 \pm$ 90). Se puede apreciar el intervalo temporal en los que pudo producirse la construcción del monumento.

\subsection{La dimensión cronológica de Los Zumacales en la secuencia del megalitismo del interior de la península ibérica}

La segunda de las interpretaciones cronológicas planteadas es más consistente con la hipótesis de ciclos funerarios breves y se adecúa mejor a la asignación de Los Zumacales a los primeros momentos de actividad funeraria megalítica en la Meseta Norte -primera mitad del IV milenio cal AC-según las dataciones absolutas sobre hueso humano. Comportamientos similares tendrían sepulcros colectivos como El Alto del Reinoso (Burgos), con una secuencia funeraria de no más de tres generaciones, probablemente entre 3770 y 3539 cal AC como intervalo de probabilidad máximo (2б) (Alt et al. 2016: 6), las tumbas sorianas de La Sima II (Rojo et al. 2005) y La Mina (Rojo et al. 2015) o el túmulo burgalés del Silo (Moreno et al. 2012) con fechas muy similares a los primeros enterramientos de Los Zumacales (KIA-21551 $4919 \pm 28$ BP, Beta-316132 $4970 \pm 30$ BP y GrA-38105 $4955 \pm$ 33, respectivamente).

Dentro del ámbito meseteño, las únicas dataciones más antiguas proceden de la tumba paradolménica de El Miradero, también vallisoletana (Delibes et al. 1987), de algunos sepulcros del conjunto megalítico burgalés de la Lora (Delibes y Rojo 1997) y de dos yacimientos sorianos: el citado túmulo de la Sima en Miño de Medinaceli (ahora en su horizonte inicial) y el sepulcro de La Tarayuela (Rojo et al. 2005). Recordemos que, en su mayoría, son dataciones a partir de muestras de carbón y que aquellas que se remontan a fines del $\mathrm{V}$ milenio cal $\mathrm{AC}$ proceden de horizontes infratumulares o fases de construcción de los sepulcros. Por tanto, la secuencia funeraria del dolmen de Los Zumacales se correspondería casi en su totalidad con las fases iniciales del megalitismo de la Meseta Norte.

Otros focos megalíticos del interior peninsular arrojan fechas radiocarbónicas ilustrativas de una implantación muy temprana del megalitismo. En el Valle del Tajo, sendas dataciones sobre hueso humano del dolmen de Azután (Ly $45785750 \pm 130 \mathrm{BP}$ ) y del túmulo del Castillejo (Beta $1329175710 \pm 150$ BP) apuntan a la plenitud del V milenio cal AC (Bueno 1991; Bueno et al. 1999, Bueno et al. 2016). Se las une otra más propia de los inicios del IV milenio cal AC en Portillo de las Cortes (Bueno et al. 2016). La Rioja Alavesa es otra estación megalítica tradicionalmente vinculada a los dólmenes meseteños. Comparten arquitectura, ajuares y elementos simbólicos como los ídolos-espátula de tipo San Martín-El Miradero (Delibes et al. 1987; Fernández Eraso et al. 2015). Sin embargo solo un par de fechas remiten al final del V milenio: una del nivel de base del sepulcro camerano de Collado Palomero II (CSIC-897 $4900 \pm 110$ BP) (López de Calle e Ilarraza 1997) y otra del dolmen de la Chabola de la Hechicera (Beta-307795 $4940 \pm 30$ BP) (Fernández-Eraso y Mújika 2013: 99). Los investigadores de los megalitos de esta zona no descartan que la ausencia de dataciones de esta primera etapa se deba a problemas de conservación o a cuestiones culturales y funcionales, tales como vidanges o limpiezas periódicas de los osarios. 


\subsection{El último enterramiento prehistórico de Los Zumacales en el contexto del Calcolítico Inicial normeseteño}

La fecha prehistórica más moderna obtenida en nuestro proyecto (Poz-93612 $4410 \pm 35 \mathrm{BP} ; 3321$ 2915 cal $2 \sigma \mathrm{AC}$ ) indicaría un uso del sepulcro de Los Zumacales más allá del Neolítico Final, ya en plena época calcolítica, si asumimos que no es un valor atípico por desviaciones en la curva de calibración, contaminación o reservorio isotópico anómalo (Bronk Ramsey 2009b). En ello incide, por otro lado, que la tibia fechada fuera recuperada en las cotas superiores del osario (véase Tab. 3). La falta de ajuares funerarios evolucionados corroboraría la condición esporádica de este enterramiento al no poder identificar una actividad funeraria más sólida en torno a estas fechas, o bien sugeriría la alteración de los valores de las fechas BP por otra serie de razones. El que en el yacimiento las únicas evidencias, ya comentadas, sean el ajuar muy homogéneo y las pocas inhumaciones justifica que desde la perspectiva más estrictamente arqueológica siempre se haya apostado por el abandono del dolmen tras una breve utilización (Alonso Díez et al. 2015).

El Calcolítico se identifica en el registro arqueológico de la Meseta Norte a partir del c. $3100 \mathrm{cal}$ AC y se subdivide en Inicial o Precampaniforme (3100$2400 \mathrm{cal} \mathrm{AC})$ y Campaniforme (2400-2000 cal AC). Este periodo supuso la adopción de grandes transformaciones en los modos de poblamiento, la economía y la organización social (Delibes 2011; Villalobos 2016). En la dimensión de las prácticas funerarias implicó el abandono de los dólmenes y la generalización de fórmulas funerarias como las inhumaciones en hoyo (Fabián 1995). Los dólmenes tardoneolíticos, por su condición de hitos territoriales tanto como de construcciones monumentales, fueron elementos todavía muy presentes en el paisaje en el que se desarrollaron las comunidades calcolíticas. Es bastante habitual encontrar en ellos artefactos propios del ajuar campaniforme (Delibes y Santonja 1986). De modo excepcional, caso de La Sima (Rojo et al. 2005), formaban parte de auténticos enterramientos intrusivos. La cuestión es más compleja respecto a los materiales calcolíticos precampaniformes. Se conocen materiales de este horizonte en muchos dólmenes salmantinos y zamoranos (Arias 1989; Palomino 1990; López Plaza et al. 2000) y, al menos, hay un par de casos donde participan en eventos de clausura (Villalobos 2015; Tejedor et al. 2017). En cambio, prácticamente no hay pruebas de inhumaciones calcolíticas precampaniformes en dólmenes. La síntesis exhaustiva de C. Tejedor (2015: cap. 6.3) sólo ha identificado el caso de Arroyal I (Carmona 2014), al que añadimos la datación c. 3300-2900 cal AC sobre hueso humano de La Velilla (Delibes et al. 2012), otro redondil, cuyas puntas de flecha de retoque plano (Zapatero 1989) ya sugerían una reutilización en estas fechas.

De esta manera nuestro pretendido inhumado calcolítico precampaniforme de Los Zumacales, un dolmen sin artefactos documentados de esta época (Alonso Díez et al. 2015), de ser correcta la datación, o es una rareza o, quizás, la primera prueba contundente de un comportamiento, hasta ahora, con escasa visibilidad arqueológica. Anteriormente hemos vinculado la llegada del Calcolítico a la Meseta Norte con la adopción de inhumaciones en hoyo a veces acompañadas de artefactos sociotécnicos de prestigio como útiles de metal y adornos de variscita y otros minerales de vivos colores. Junto a estas tumbas "ricas", se calcula que hay entre un $75 \%$ y un $92 \%$ de inhumados sin ajuar o con un cuenco y poco más (Villalobos 2016: 165). Esta asimetría en la representación funeraria suele entenderse como prueba de una sociedad de rango o, incluso, de clases. Desde este punto de vista nuestro inhumado calcolítico de Los Zumacales era una persona perteneciente al común de la población que era enterrada con escaso o ningún ajuar.

Hasta la fecha las reutilizaciones calcolíticas de los dólmenes se han interpretado como intentos de la nueva élite social de legitimar su posición vinculándose artificialmente con "los ancestros". La hipótesis está bastante bien fundamentada para el caso de la sociedad Campaniforme donde artefactos sociotécnicos de prestigio como el set cerámico, las armas de cobre, los adornos de oro, etc. mostraban el alto estatus de los "intrusos" (Delibes y Santonja 1986; Rojo et al. 2005). Pero esto solamente sirve para ese segundo periodo de la Edad del Cobre. La aparición de un inhumado sin ajuar en los albores del Calcolítico Inicial, tres siglos después de finalizar la utilización original de la tumba, podría ser una prueba de que, en estos momentos, los dólmenes no serían únicamente escenario, p. ej., de ceremonias de clausura sino también de inhumaciones de ¿gente del común? ¿marginados, incluso? A $20 \mathrm{~km}$ al noreste de Los Zumacales las tres mujeres del Calcolítico Inicial, probablemente ajusticiadas en Los Cercados y que se han vinculado con un sacrificio ritual de tipo propiciatorio (García Barrios 2007), no dejan de mostrar una violencia descarnada contra ciertos miembros de la sociedad. Resulta imposible certificar que el inhumado calcolítico de Los Zumacales fuera apartado del ritual funerario más aceptado entre sus congéneres por circunstancias socioeconómicas, pero apuntamos esta posibilidad para su contraste futuro en proyectos de dataciones exhaustivas, similares a éste. 


\section{CONCLUSIONES}

Este trabajo ofrece la primera secuencia radiocarbónica de un dolmen de la Meseta obtenida a partir de dataciones sobre hueso humano del número mínimo de enterrados. Hasta ahora la investigación de la temporalidad del megalitismo se nutría predominantemente de fechas absolutas sobre carbón, ya fueran infratumulares o camerales, se corría siempre el riesgo de que las dataciones de los eventos de la vida del mo-

\begin{tabular}{|c|c|c|c|c|c|}
\hline Yacimiento & $\begin{array}{c}\text { Ref. } \\
\text { laboratorio }\end{array}$ & Fechas BP & $\begin{array}{c}\text { Datación calibrada } \\
1 \sigma \text { cal AC }\end{array}$ & $\begin{array}{c}\text { Datación calibrada } \\
2 \sigma \text { cal AC }\end{array}$ & Referencia \\
\hline Azután (Toledo) & Ly 4578 & $5750 \pm 130$ & $4766-4456$ & $4904-4345$ & Bueno, 1991 \\
\hline El Castillejo (Toledo) & Beta 132917 & $5710 \pm 150$ & $4716-4373$ & $4936-4263$ & Bueno et al., 1999 \\
\hline $\begin{array}{l}\text { San Juan Ante Portam } \\
\text { Latinam (Álava) }\end{array}$ & I-14842* & $5070 \pm 150$ & $4037-3696$ & $4253-3536$ & Vegas et al., 1999 \\
\hline Azután (Toledo) & UGRA 288 & $5060 \pm 90$ & $3962-3770$ & $4039-3657$ & Bueno, 1991 \\
\hline Zumacales (Valladolid) & Poz-93536 & $5020 \pm 40$ & $3936-3715$ & 3944-3709 & Presente estudio \\
\hline $\begin{array}{l}\text { San Juan Ante Portam } \\
\text { Latinam (Álava) }\end{array}$ & I-14594* & $5020 \pm 140$ & $3959-3666$ & $4228-3522$ & Vegas et al., 1999 \\
\hline Zumacales (Valladolid) & Poz-93542 & $5010 \pm 40$ & $3923-3712$ & $3944-3704$ & Presente estudio \\
\hline $\begin{array}{l}\text { Portillo de las Cortes } \\
\text { (Guadalajara) }\end{array}$ & Beta 334952 & $5000 \pm 30$ & $3892-3712$ & $3939-3702$ & Bueno et al., 2016 \\
\hline Zumacales (Valladolid) & Poz-93537 & $4990 \pm 40$ & 3894-3706 & $3941-3661$ & Presente estudio \\
\hline Zumacales (Valladolid) & Poz-93543 & $4990 \pm 35$ & $3797-3709$ & $3938-3664$ & Presente estudio \\
\hline Zumacales (Valladolid) & Poz-93538 & $4990 \pm 35$ & $3797-3709$ & $3938-3664$ & Presente estudio \\
\hline Zumacales (Valladolid) & Poz-93611 & $4975 \pm 35$ & $3787-3706$ & $3924-3658$ & Presente estudio \\
\hline La Mina (Soria) & Beta-316132 & $4970 \pm 30$ & $3777-3707$ & 3893-3661 & Rojo et al., 2015 \\
\hline El Silo (Burgos) & GrA-38105 & $4955 \pm 30$ & $3772-3698$ & $3790-3658$ & $\begin{array}{l}\text { Moreno et al., } \\
\text { 2010-2012 }\end{array}$ \\
\hline $\begin{array}{l}\text { Chabola de La } \\
\text { Hechicera (La Rioja) }\end{array}$ & Beta-307795 & $4940 \pm 30$ & $3761-3661$ & $3777-3654$ & $\begin{array}{c}\text { Fernández-Eraso y } \\
\text { Mújika-Alustiza, } 2013\end{array}$ \\
\hline $\begin{array}{l}\text { El Alto del Reinoso } \\
\text { (Burgos) }\end{array}$ & $\begin{array}{c}\text { MAMS- } \\
14327 \\
\end{array}$ & $4933 \pm 27$ & $3748-3658$ & $3770-3653$ & Rojo et al., 2015 \\
\hline La Sima II (Soria) & KIA-21551 & $4919 \pm 28$ & $3706-3657$ & $3766-3646$ & Rojo et al., 2005 \\
\hline $\begin{array}{l}\text { El Alto del Reinoso } \\
\text { (Burgos) }\end{array}$ & $\begin{array}{l}\text { MAMS- } \\
14325\end{array}$ & $4911 \pm 25$ & $3700-3657$ & $3761-3643$ & Rojo et al., 2015 \\
\hline $\begin{array}{l}\text { Collado Palomero II } \\
\text { (La Rioja) }\end{array}$ & CSIC-897 & $4900 \pm 110$ & $3907-3532$ & $3956-3381$ & $\begin{array}{c}\text { López e Ilarraza, } \\
1997\end{array}$ \\
\hline Zumacales (Valladolid) & Poz-93610 & $4895 \pm 35$ & $3697-3649$ & $3762-3637$ & Presente estudio \\
\hline La Sima II (Soria) & KIA-21553 & $4865 \pm 23$ & $3662-3638$ & $3699-3636$ & Rojo et al., 2005 \\
\hline La Sima II (Soria) & KIA-21552 & $4862 \pm 27$ & $3691-3638$ & $3702-3545$ & Rojo et al., 2005 \\
\hline $\begin{array}{l}\text { El Alto del Reinoso } \\
\text { (Burgos) }\end{array}$ & $\begin{array}{c}\text { MAMS- } \\
14326 \\
\end{array}$ & $4854 \pm 26$ & $3661-3636$ & $3698-3539$ & Rojo et al., 2015 \\
\hline La Sima II (Soria) & KIA-21550 & $4839 \pm 27$ & $3655-3541$ & $3695-3533$ & Rojo et al., 2005 \\
\hline Zumacales (Valladolid) & Poz-112689 & $4820 \pm 35$ & $3650-3536$ & $3692-3522$ & Presente estudio \\
\hline
\end{tabular}

Tab. 4. Fechas C14 sobre hueso humano correspondientes a megalitos del interior de la península ibérica (finales del V milenio-principios de IV milenio cal AC).

* Conviene advertir que estas dataciones han sido recientemente rechazadas para los estudios de temporalidad realizados sobre el hipogeo de San Juan Ante Porta Latinam (Laguardia, Álava). Esto se debe a alto valor de desviación típica y al desfase temporal con respecto al conjunto de fechas obtenidas en el yacimiento (Fernández-Crespo et al. 2018: 3).

Trab. Prehist., 77, N. ${ }^{\circ}$ 1, enero-junio 2020, pp. 130-147, ISSN: 0082-5638

https://doi.org/10.3989/tp.2020.12250 
numento se vieran afectados por el "síndrome de madera vieja", y se situaban los inicios del megalitismo meseteño entre los últimos siglos del $\mathrm{V}$ milenio cal AC y los inicios del IV milenio cal AC.

Según los resultados de la datación de nueve individuos del dolmen de Los Zumacales en su mayoría fueron inhumados en el lapso c. 3900-3600 cal AC $(2 \sigma)$. Ello estaba en relativa consonancia con las dataciones, también en la primera mitad del IV milenio cal AC, de gran parte de los huesos de otros megalitos de la Meseta Norte y del Valle del Tajo: La Mina y La Sima (Soria), El Alto del Reinoso y El Silo (Burgos), Azután (Toledo) y El Portillo de las Cortes (Guadalajara). Además, contamos con una solitaria datación en c. 3300-2900 cal AC $(2 \sigma)$ que se corresponde con ciertas fechas de la segunda mitad del IV milenio cal $\mathrm{AC}$ en sepulcros burgaleses como Las Arnillas, palentinos como La Velilla y toledanos como El Castillejo y el túmulo del Valle de las Higueras. Este comportamiento difiere de lo que se observa, p. ej., en el Valle del Ebro a su paso por La Rioja y Âlava. Allí, pese a haber huesos fechados en la primera mitad del IV milenio cal AC (Collado Palomero II, La Chabola de la Hechicera), las dataciones, en general, se concentran en la segunda mitad de dicho milenio, prolongándose incluso durante las Edades del Cobre y Bronce. A su vez en el sureste peninsular, se registra un uso continuado de los sepulcros desde el segundo tercio del IV milenio cal AC hasta finales del III milenio cal AC.

La aplicación del análisis bayesiano a la serie de Los Zumacales muestra la robustez estadística de la agrupación de los individuos en ese primer y relativamente breve periodo entre 3857-3711 cal AC y 37763636 cal AC $(2 \sigma)$ que podría durar hasta 130 años y la posible existencia de un segundo evento de reutilización funeraria puntual, ya durante el Calcolítico c. $3300-2900$ cal AC $(2 \sigma)$. La conclusión que extraemos del primer periodo de uso de Los Zumacales es que el monumento fue construido, utilizado y amortizado en un breve lapso de tiempo. Ello está muy lejos de la idea del megalito como una "tumba para la eternidad" de utilización diacrónica y recurrente. Planteamos a partir del segundo uso, y en adelante, que la total ausencia de ajuar propio del Calcolítico Inicial nos sitúa ante el uso del dolmen no por una élite ansiosa de legitimar su posición social en los monumentos del pasado sino, por el contrario, por el entorno de una persona perteneciente al común o, incluso, un marginado social. Por último, no debemos olvidar la probable inclusión en la serie funeraria de dos individuos de época medieval (siglos X-XII DC), pese a la ausencia en Los Zumacales de elementos materiales propios de esta cronología.

Finalmente, insistimos en los dobles resultados de la nueva metodología de trabajo, consistente en datar casi la totalidad del número mínimo de individuos recuperados en los sepulcros colectivos. Además de ofrecer respuestas rotundas a viejos interrogantes sobre el fenómeno megalítico, muestra hechos que permiten el planteamiento de nuevas preguntas que deberán ser atendidas en el futuro.

\section{AGRADECIMIENTOS}

La Dirección General de Patrimonio Cultural de la Junta de Castilla y León asumió el gasto de las dataciones radiocarbónicas en las que se basa el artículo. Jesús del Val y Nicolás Benet (Servicio de Planificación y Estudios, DGPC de la JCyL) nos facilitaron la tramitación de la ayuda. El profesor Carlos Matrán Bea (Dpto. de Estadística e Investigación Operativa, Universidad de Valladolid) nos orientó en la redacción del trabajo.

\section{BIBLIOGRAFÍA}

Alonso Díez, M.; Delibes de Castro, G. y Santiago Pardo, J. 2015: "El sepulcro megalítico de Los Zumacales, en Simancas (Valladolid)". En E. Wattenberg García (ed.): Conocer Valladolid 2014. VIII Curso de Patrimonio Cultural. Real Academia de Bellas Artes de la Purísima Concepción. Valladolid: 13-35.

AlQahtani, S. J.; Hector, M. P. y Liversidge, H. M. 2010: "Brief communication: the London atlas of human tooth development and eruption". American Journal of Physical Anthropology 142 (3): 481-490.

Alt, K. W.; Zesch, S.; Garrido-Pena, R.; Knipper, C.; Szécsényi-Nagy, A.; Roth, C., ... and Rojo-Guerra, M. A. 2016: "A community in life and death: The late neolithic megalithic tomb at Alto de Reinoso (Burgos, Spain)". PLOS ONE 11 (1). https://doi.org/10.1371/journal.pone.0146176

Aranda Jiménez, G. y Lozano Medina, A. 2014: "The chronology of megalithic funerary practices: A Bayesian approach to Grave $11 \mathrm{At}$ El Barranquete necropolis (Almería, Spain)". Journal of Archaeological Science 50 (1): 369-382. https://doi.org/10.1016/j.jas.2014.08.005

Aranda Jiménez, G., Lozano Medina, Á., Camalich Massieu, M. D., Martín Socas, D., Rodríguez Santos, F. J., Trujillo Mederos, A... y Clop García, X. 2017: "La cronología radiocarbónica de las primeras manifestaciones megalíticas en el sureste de la Península Ibérica: las necrópolis de Las Churuletas, La Atalaya y Llano del Jautón (Purchena, Almería)". Trabajos de Prehistoria 74 (2): 257-277. https://doi.org/10.3989/tp.2017.12194

Arias González, L. 1989: "Contribución al estudio del Fenómeno Megalítico en el Occidente de la Meseta Norte: el dolmen de 'El Torrejón' (Villarmayor, Salamanca)". En XIX Congreso Nacional de Arqueología (Valencia 1987): 399-408. Zaragoza.

Bayliss, A.; Ramsey, C. B.; Van Der Plicht, J. y Whittle, A. 2007: "Bradshaw and bayes: towards a timetable for the neolithic". Cambridge Archaeological Journal 17 (SUPPL.1): 1-28. https://doi.org/10.1017/S0959774307000145

Bocquet-Appel, J-P. y de Miguel Ibáñez, M. P. 2002: "Demografía de la difusión neolítica en Europa y los datos paleoantropólogicos". Saguntum Extra 5: 23-44.

https://ojs.uv.es/index.php/saguntumextra/article/download/10692/9890.

Bronk Ramsey, C. 1995: "Radiocarbon calibration and analysis of stratigraphy: the OxCal Program". Radiocarbon 37 (02): 425-430. https://doi.org/10.1017/S0033822200030903 
Bronk Ramsey, C. 2001: "Development of the radiocarbon calibration program". Radiocarbon 43 (2): 355-363.

Bronk Ramsey, C. 2009a: "Bayesian analysis of radiocarbon dates". $R a$ diocarbon 51 (1): 337-360.

Bronk Ramsey, C. 2009b: "Dealing with outliers and offsets in radiocarbon dating". Radiocarbon 51 (3): 1023-1045. https://doi.org/10.1017/S0033822200034093

Brothwell, D. R. 1981: Digging up bones: the excavation, treatment, and study of human skeletal remains. Cornell University Press. New York.

Bueno Ramírez, P. 1991: Megalitos en la Meseta sur: los dólmenes de Azután y La Estrella, Toledo. Ministerio de Cultura. Madrid.

Bueno Ramírez, P.; Balbín Behrmann, R. de; Barroso Bermejo, R. 2016 "Between east and west: megaliths in the centre of the Iberian Peninsula". En L. Laporte y Ch. Scarre (eds.): The megalithic architectures of Europe. Oxbow books. Oxford: 157-166.

Bueno Ramírez, P.; Balbín Behrmann, R. de; Barroso Bermejo, R.; Rojas Rodríguez-Malo, J. M.; Villa González, R.; Félix López, R. y Rovira Llorens, S. 1999: "Neolítico y Calcolítico en Huecas (Toledo) El Túmulo de Castillejo. Campaña de 1998". Trabajos de Prehistoria 56 (2): 141-160.

Buikstra, J. E. y Ubelaker, D. H. 1994: Standards for data collection from human skeletal remains, Proceedings of a Seminar at the Field Museum of Natural History. Arkansas Archaeological Survey Research Series 44, Arkansas Archaeological Survey Press. Fayetteville.

Carmona Ballestero, E.; Arnaiz Alonso, M. Á. y Alameda Cuenca-Romero, M. C. 2014: "El dolmen de Arroyal I: usos y modificaciones durante el III milenio cal AC". En J. Honrado, M. Á. Brezmes, A. Tejeiro y Ó. Rodríguez (eds.): II Jornadas de Jóvenes Investigadores del Valle del Duero. Del Neolítico a la Antigüedad Tardía (León 2012) 2. Glyphos Publicaciones. Valladolid: 41-54.

Delibes de Castro, G. 1984: "Fechas de radiocarbono para el Megalitismo de la Meseta Española”. Arqueología. Grupo de Estudos Arqueologicos do Porto 10: 99-102.

Delibes de Castro, G. 1995: "Ritos funerarios, demografía y estructura social entre las comunidades neolíticas de la submeseta norte". En R. Fábregas, F. Pérez y C. Fernández (eds.): Arqueología da Morte. Arqueología da Morte na Península Ibérica desde as Orixes ata o Medievo (Xinzo de Lima 1995): 61-94. Concello de Xinzo de Limia.

Delibes de Castro, G. 2000: "Del Bronce al Hierro en el Valle Medio del Duero: una valoración del límite Cogotas I - Soto de Medinilla a partir de las manifestaciones de culto". Zephyrus 53-54: 293-309.

Delibes de Castro, G. 2010: "La investigación de las sepulturas colectivas monumentales del IV milenio A.C. en la Submeseta Norte española. Horizonte 2007”. En J. Fernández Eraso y J. A. Mujika-Alustiza (eds.): Actas del Congreso Internacional sobre Megalitismo y otras manifestaciones funerarias contemporáneas en su contexto social, económico y cultural. Munibe. Suplemento 32, Sociedad de Ciencias Aranzadi. Donostia: 12-56.

Delibes de Castro, G. 2011: El pan y la sal. La vida campesina en el Valle Medio del Duero hace cinco mil años. Real Academia de Bellas Artes de la Purísima Concepción. Valladolid.

Delibes de Castro, G.; Alonso Díez, M. y Rojo Guerra, M. A. 1987 "Los sepulcros colectivos del Duero Medio y Las Loras, y su conexión con el foco dolménico riojano". En El Megalitismo en la Península Ibérica. Ministerio de Cultura. Madrid: 181-197.

Delibes de Castro, G. y Etxeberría Gabilondo, F. 2002: "Fuego y cal en el sepulcro colectivo de "El Miradero" (Valladolid): ¿accidente, ritual o burocracia de la muerte?" En M. A. Rojo Guerra y M. Kunst (eds.): Sobre el significado del Fuego en los Rituales Funerarios del Neolítico. Secretariado de Publicaciones e Intercambio Editorial. Valladolid: 39-55.

Delibes de Castro, G.; Guerra Doce, E.; Zapatero Magdaleno, P. y Villalobos García, R. 2012: "Les spatules-idoles de type San Martín-El Miradero: Identité, symbolisme, liturgie et prestige dans les mobiliers des tombes mégalithiques de la Vielle Castille (Espagne)". En M. Sohn y J. Vaquer (eds.): Sépultures collectives et mobiliers funéraires de la fin du Néolitique en Europe occidentale: 305-331. Archives d'écologie préhistorique.

Delibes de Castro, G. y Rojo Guerra, M. A. 1997: “C14 y secuencia megalítica en La Lora burgales: acotaciones a la problemática de las dataciones absolutas referentes a yacimientos dolménicos”. En A. Rodrígez Casal (ed.): O Neolítico atlántico e as orixes do megalitismo. Universidad de Santiago de Compostela. Santiago de Campostela: $391-414$

Delibes de Castro, G. y Rojo Guerra, M. 2002: "Reflexiones sobre el trasfondo cultural del polimorfismo megalítico en la Lora burgalesa". Archivo Español de Arqueología 75: 21-35.

Delibes de Castro, G.; Rojo Guerra, M. Á. y Represa Bermejo, J. I. 1993: Dólmenes de La Lora. Burgos. Junta de Castilla y León. Valladolid.

Delibes de Castro, G. y Santonja Gómez, M. 1986: El fenómeno megalítico en la provincia de Salamanca. Ediciones de la Diputación de Salamanca. Salamanca.

Fabián García, J. F. 1995: El aspecto funerario durante el Calcolítico y los inicios de la Edad del Bronce en la Meseta Norte. Ediciones de la Universidad de Salamanca. Salamanca.

Ferembach, D.; Schwidetzky I y Stloukal M. 1980: "Recomendations for age and sex diagnosis of skeletons". Journal of human evolution 9: 517-549.

Fernández-Crespo, T. y de la Rúa, C. 2015: "Demographic evidence of selective burial in megalithic graves of northern Spain”. Journal of Archaeological Science, 53: 604-617. https://doi.org/10.1016/j.jas.2014.11.015

Fernández-Crespo, T.; Schulting, R. J.; Ordoño, J.; Duering, A.; Etxeberria, F.; Herrasti, L., ... y Bronk Ramsey, C. 2018: "New radiocarbon dating and demographic insights into San Juan ante Portam Latinam, a possible Late Neolithic war grave in North-Central Iberia". American journal of physical anthropology 166 (3): 760-771. https://doi.org/10.1002/ajpa.23465

Fernández-Eraso, J. y Mujika-Alustiza, J. A. 2013: "The megalithic station of the Rioja Alavesa: chronology, origins and utilisation cycles". Zephyrus 71: 89-106.

Fernández-Eraso, J.; Mujika-Alustiza, J. A. y Fernández-Crespo, T. 2015: "Sobre la cronología de los ídolos-espátula del dolmen de San Martín (Laguardia-Álava)". ARPI, Arqueología y Prehistoria del Interior Peninsular 3: 257-271.

García Barrios, A. S. 2007: "Un enfoque de género en la arqueología de la Prehistoria Reciente del valle medio del Duero: los cráneos femeninos calcolíticos de Los Cercados (Mucientes, Valladolid)". En C. de la Rosa, M. J. Dueñas, M. I. del Val y M. Santo Tomás (eds.): Nuevos enfoques para la enseñanza de la historia. Mujer y género ante el espacio europeo de educación superior. Asociación Cultural Al-Mudayna. Madrid: 41-56

García Sanjuán, L.; Wheatley, D. W. y Costa Caramé, M. E. 2011: "The numerical chronology of the megalithic phenomenon in southern Spain: progress and problems". Menga (1): 121-158.

Guerra Doce, E.; Delibes de Castro, G.; Zapatero Magdaleno, P. y Villalobos García, R. 2009: "Primus inter pares: estrategias de diferenciación social en los sepulcros megalíticos de la Submeseta Norte Española". Boletín del Seminario de Arte y Arqueología. Arqueología 75: 41-65.

Jiménez-Brobeil, S. A. 2012: "Patrones de actividad en el pasado. Comentario sobre algunos métodos de estudio y sus posibilidades". Cuadernos de Prehistoria y Arqueología de la Universidad de Granada 22: 91-110.

Jiménez-Brobeil, S. A., Oumaoui, I. A. y Souich, P. 2010: "Some types of vertebral pathologies in the Argar culture (Bronze Age, SE Spain)". International Journal of Osteoarchaeology 20 (1): 36-46.

López de Calle, C. e Ilarraza, J. A. 1997: "Fases Antiguas del megalitismo de Cameros (La Rioja): caracterización y cronología”. En A. Rodrígez Casal (eds.): $O$ Neolítico atlántico e as orixes do megalitismo. Universidad de Santiago de Compostela. Santiago de Campostela: $415-428$.

López Plaza, S.; Luis Francisco, J. y Salvador Mateos, R. 2000: “Megalitismo y vías de comunicación en el SO Salmantino". En V. O. Jorge (ed.): Actas do $3^{\circ}$ Congresso de Arqueología Peninsular. PréHistoria Recente da Península Ibérica (Porto 2000): 271-288. Porto

Lovejoy, C. O. 1985: "Dental wear in the Libben population: its functional pattern and role in the determination of adult skeletal age at death". American Journal of Physical Anthropology 68 (1): 47-56.

Trab. Prehist., 77, N. ${ }^{\text {o }} 1$, enero-junio 2020, pp. 130-147, ISSN: 0082-5638

https://doi.org/10.3989/tp.2020.12250 
Lovejoy, C. O.; Meindl, R. S.; Pryzbeck, T. R. y Mensforth, R. P. 1985: "Chronological metamorphosis of the auricular surface of the ilium: a new method for the determination of adult skeletal age at death". American journal of physical anthropology 68 (1): 15-28.

Lozano Medina, Á. y Aranda Jiménez, G. 2018: "Long-lasting sacred landscapes: the numerical chronology of the megalithic phenomenon in south-eastern Iberia”. Journal of Archaeological Science: Reports 19 (February): 224-238. https://doi.org/10.1016/j.jasrep.2018.02.038

Martín-Gil, J.; Martín-Gil, F. J.; Delibes de Castro, G.; Zapatero Magdaleno, P. y Sarabia Herrero, F. J. 1995: "The first known use of vermillion". Cellular and Molecular Life Sciences 51 (8): 759-761.

Masset, C. 1986: "Le recrutement d'un ensemble funéraire". En H. Duday y C. Masset (eds.): Anthropologie physique et archéologie: méthodes d'études des sépultures. Editions du CNRS. Paris: 111-134.

Meindl, R. S.; Lovejoy, C. O.; Mensforth, R. P. y Walker, R. A. 1985: "A revised method of age determination using the os pubis, with a review and tests of accuracy of other current methods of pubic symphyseal aging". American Journal of Physical Anthropology 68 (1): 29-45.

Morán Bardón, C. 1931: Excavaciones en los dólmenes de Salamanca. Junta Superior de Excavaciones y Antigüedades. Madrid

Moreno Gallo, M. Á. 2004: Megalitismo y geografia. Análisis de los factores de localización espacial de los Dólmenes de la provincia de Burgos. Universidad de Valladolid. Valladolid.

Moreno Gallo, M. A.; Delibes de Castro, G.; López Sáez, J. A.; Manzano Rodríguez, S.; Villalobos García, R.; Fraile Vicente, A. y Bastoncillos Arce, J. 2010-2012: "Nuevos datos sobre una alineación de menhires en el norte de Burgos: el yacimiento de Las Atalayas, en Avellanosa del Páramo (Burgos)". Sautuola 16-17: 71-93.

Palomino Lázaro, Á. L. 1990: "Nuevas aportaciones al conocimiento del fenómeno megalítico de la provincia de Zamora". En I Congreso de Historia de Zamora (Zamora 1990) II: 173-200. Zamora.

Reimer, P. J.; Bard, E.; Bayliss, A.; Beck, J. W.; Blackwell, P. G.; Bronk Ramsey, C., ... y Ho, J. 2013: "IntCal13 and Marine13 radiocarbon age calibration curves, 0-50,000 years cal BP”. Radiocarbon 55 (4): 1869-1887.

Robb, J. 2016: "What can we really say about skeletal part representation, MNI and funerary ritual? A simulation approach". Journal of Archaeological Science: Reports 10: 684-692.

Rojo Guerra, M. A.; Garrido Pena, R.; Tejedor Rodriguez, C.; GarcíaMartínez de Lagrán, I.; Alt, K. W., y Zesch, S. 2015: "El tiempo y los ritos de los antepasados: La Mina y El Alto del Reinoso, novedades sobre el megalitismo en la Cuenca del Duero". ARPI. Arqueología y Prehistoria del Interior Peninsular 3: 1-15.

Rojo Guerra, M. Á.; Kunst, M.; Garrido Pena, R.; García Martínez de Lagrán, Í., y Morán Dauchez, G. 2005: Un desafio a la eternidad: Tumbas monumentales del Valle de Ambrona.Junta de Castilla y León. Valladolid.

Romón, C. 1960: "Yacimiento del Paleolítico Inferior en Arroyo-Simancas". Boletín del Seminario de Arte y Arqueología 26: 155-161

Scheuer, L. y Black, S. 2000: Developmental juvenile osteology. Academic Press.

Schiffer, M. B. 1986: "Radiocarbon dating and the "old wood" problem: the case of the Hohokam chronology". Journal of Archaeological Science 13 (1): 13-30. https://doi.org/10.1016/0305-4403(86)90024-5
Szécsényi-Nagy, A.; Roth, C.; Brandt, G.; Rihuete-Herrada, C.; Tejedor Rodríguez, C.; Held, P., ... and Alt, K. W. 2017: “The maternal genetic make-up of the Iberian Peninsula between the Neolithic and the Early Bronze Age". Scientific Reports 7 (1): 15644. https://doi.org/10.1038/s41598-017-15480-9

Tejedor Rodríguez, C. 2015: La pervivencia de los 'usos megalíticos'en el valle del Duero/Douro a lo largo de la Prehistoria reciente (IV-II milenio cal. BC). Tesis doctoral, Facultad de Letras, Universidad de Valladolid. http://uvadoc.uva.es/handle/10324/17998 (consulta 31-12020).

Tejedor, C.; Rojo, M.; Garrido, R.; García, I. y Palomino, A. 2017: “'Biografía' de un monumento megalítico: fases de uso y clausura en el dolmen de El Teriñuelo". Zephyrus 89: 39-61.

Vegas, J. I.; Armendariz, A.; Etxeberria, F.; Fernández, M. S.; Herrasti, L., y Zumalabe, F. 1999: "La sepultura colectiva de San Juan ante Portam Latinam (Laguardia, Álava)". Saguntum Extra 2, II Congrés del Neolític a la Península Ibèrica: 439-445.

Villalobos García, R. 2015: "The megalithic tombs of the Spanish Northern Meseta. Material, political and ideological ties between the Neolithic people and their territory". Préhistoires Méditerranéennes (Colloque 2014): 1-17. http://journals.openedition.org/pm/1047

Villalobos García, R. 2015: Análisis de las transformaciones sociales en la Prehistoria reciente de la Meseta Norte española (milenios VI-III $C A L A C)$ a través de empleo de la variscita y otros minerales verdes como artefactos sociotécnicos. Tesis doctoral, Universidad de Valladolid. Facultad de Filosofía y Letras. https://doi.org/10.35376/10324/16693

Villalobos García, R. 2016: Análisis de las transformaciones sociales en la Prehistoria Reciente de la Meseta Norte española (milenios VI-III cal a.C.). Studia Archaeologica 101, Ediciones Universidad de Valladolid. Valladolid.

Villalobos García, R.; Odriozola, C. P.; Delibes de Castro, G.; Santonja Gómez, M.; Pérez Martín, R.; Benet Jordana, N.; Fabián, J. F.; Guerra Doce, E. y Zapatero Magdaleno, P. 2018: "Cadena operativa y análisis tecno-tipológico de los adornos prehistóricos de variscita del centro-sur-occidente de la Meseta Norte Española. Historia de una tradición artesanal". Complutum 29 (1): 59-78. https://doi.org/10.5209/CMPL.62395

Ward, G. K. y Wilson, S. R. 1978: "Procedures for comparing and combining radiocarbon age determinations: a critique". Archaeometry 20 (1): 19-31. https://doi.org/10.1111/j.1475-4754.1978.tb00208.x

White, T. D.; Black, M. T. y Folkens, P. A. 2012: Human osteology. Academic press.

Zapatero Magdaleno, P. 1989: "La Velilla, un enterramiento de tradición dolménica en el Valle del Valdavia”. Publicaciones de la Institución Tello Téllez de Meneses 60: 9-13.

Zapatero Magdaleno, P. 1991: "Sobre las relaciones entre Neolítico interior y megalitismo: notas sobre el túmulo de La Velilla, en Osorno (Palencia)". Boletín del Seminario de Estudios de Arte y Arqueología 57: 53-61.

Zapatero Magdaleno, P. 2012: "El sepulcro de La Velilla, en Osorno (Palencia), dentro del marco del fenómeno megalítico de la Meseta Norte". Patrimonio Histórico de Castilla y León 46: 51-58. 